NASA Contractor Report 3019

\title{
A Visual Investigation of Turbulence in Stagnation Flow About a Circular Cylinder
}

Willy Z. Sadeh and Herbert J. Brauer

GRANT NSG-3127

OCTOBER 1978 
NASA Contractor Report 3019

A Visual Investigation of Turbulence in Stagnation Flow About a Circular Cylinder

Willy Z. Sadeh and Herbert J. Brauer

Colorado State University

Fort Collins, Colorado

Prepared for

Lewis Research Center

under Grant NSG-3127

N/SA

National Aeronautics

and Space Administration

Scientific and Technical

Information Office

1978 
t. 
1. INTRODUCTION 2

2. EXPERIMENTAL SETUP

3. EXPERIMENTAL PROCEDURE 9

4. EXAMINATION OF FLOW EVENTS 12

4.1 Side Views 14

4.2 Top Views 17

4.3 Rear Views $\quad 22$

5. DISCUSSION OF RESULTS 26

6. CONCLUSIONS 29

APPENDIX A - LIST OF SYMBOLS 30

REFERENCES

$\begin{array}{ll}\text { FIGURES } & 34\end{array}$ 
A VISUAL INVESTIGATION OF TURBULENCE IN STAGNATION FLOW ABOUT A CIRCULAR CYLINDER

by Willy z. Sadeh ${ }^{I}$ and Herbert J. Brauer ${ }^{2}$ Fluid Mechanics and Wind Engineering Program Department of Civil Engineering Colorado State University

SUMMARY

A visualization study of stagnation flow round a circular cylinder was carried out in order to gain a physical insight into its model put forward by the vorticity-amplification theory. The visualization was conducted at a Reynolds number of $8 \times 10^{3}$ utilizing white smoke composed of titanium dioxide for an approaching flow containing turbulence at scales larger than the neutral wavelength. An in-depth dynamic qualitative apperception of the flow events was obtained by means of motion pictures taken from three different viewpoints. A frame by frame examination of selected movie strips led further to the acquisition of a reasonably quantitative interpretation of the gross flow structure. The analysis of the flow events focused on tracing the temporal and spatial evolution of a cross-vortex tube outlined by the entrained smoke filaments.

The visualization supplied striking evidence concerning: (1) the selective stretching of cross-vortex tubes which is responsible for the amplification of cross vorticity and, hence, of streamwise turbulence; (2) the streamwise tilting of stretched cross-vortex tubes; (3) the existence of a coherent array of vortices near the stagnation zone; (4) the interaction of the amplified vorticity with the body laminar boundary layer; and, finally, (5) the growth of a turbulent boundary layer. These are basically the flow characteristics and events advanced by the vorticity-amplification theory. It is thus apparent that the amplification of turbulence in stagnation flow around a bluff body and its effects upon the body boundary layer are satisfactorily explained by the vorticity-amplification theory.

2 Research Assistant 


\section{INTRODUCTION}

The importance of turbulence amplification in the flow approaching the stagnation zone of a bluff body is currently recognized. Results of many investigations, both experimental and theoretical, clearly attest to the strong sensitivity of both stagnation-point skin friction and heat transfer to the presence and, particularly, the nature of the oncoming turbulence. Occurrence of relatively large velocity fluctuations upstream of the stagnation zone of a circular cylinder and of an airplane strut was first reported by Piercy and Richardson (refs. I and 2). A discussion of the earlier background of this flow situation can be found in a report by Kestin and Maeder (ref. 3). Additional history on this subject is available in papers by Sutera, Maeder and Kestin (ref. 4), Sutera (ref. 5), Sadeh, Sutera and Maeder (refs. 6 and 7), and in numerous other papers (e.g., see: refs. 8-12; to mention a few). A thorough survey of this flow is not given herein since the background literature is readily accessible. The vorticity-amplification theory is, however, briefly reviewed for the sake of laying down the guidelines regarding the present visualization study.

The vorticity-amplification theory advanced by sutera et al. (ref. 4) and further extended by Sadeh et al. (ref. 6) offers a satisfactory explanation for the evolution of turbulence in flow around a bluff body and its three-dimensional effects on the stagnation-point boundary layer. This theory suggests that specific turbulent fluctuations present in the oncoming stream, no matter how small initially, are susceptible to undergoing significant amplification as they are conveyed by the mean flow toward the body stagnation zone. Selective stretching of cross-vortex filaments characteristic to any diverging stagnation flow is proposed as the mechanism responsible for the amplification of vorticity and, hence, of turbulence. The stretched vortex filaments experience simultaneous streamwise tilting as they are convected toward and past the body. Relatively high levels of turbulent energy concentration at particular scales emerge near the stagnation zone as a result of the vorticity amplification. This intensified turbulence is the agent causing the observed increases in shear stress and heat transfer at the wall. Concurrently with the amplification induced by the stretching, vorticity dissipates due to the viscous action. One of the most important findings of the vorticity-amplification theory is the dependence of both amplification and dissipation upon the spatial scale of vorticity (scale or eddy size of turbulence). At scales $\lambda$ greater than a certain neutral scale $\lambda_{0}$ the stretching dominates the flow behavior. At these scales the vorticity amplifies more rapidly than it dissipates. On the other hand, at scales smaller than the neutral one the viscous dissipation is the main effect. The neutral scale $\lambda_{0}$ (or wavelength) depends on the mean velocity deceleration rate, the freestream velocity, a 
characteristic dimension of the body and the fluid viscosity. can thus be viewed as the critical scale or critical Reynolds number insofar as the vorticity amplification is concerned.

The stretching and the tilting of the vortex filaments and their viscous diffusion are readily accounted for in the vorticity transport equation. For the flow of a viscous, incompressible fluid of constant properties the vorticity transport equation in rectangular cartesian coordinates $x_{i}(i=1,2,3)$ is

$$
\frac{D \omega_{i}}{\overline{D E}}=\omega_{j} \frac{\partial U_{i}}{\partial x_{j}}+v \frac{\partial^{2} \omega_{i}}{\partial x_{j} \partial x_{j}},
$$

where the vorticity

$$
w_{i}=\varepsilon_{i j k} \frac{\partial U_{k}}{\partial x_{j}}
$$

in which $\varepsilon_{i j k}$ is the third-order unit alternating tensor (the Levi-civita antisymmetric symbol), and $i, j, k$ take on the values $1,2,3$. In the foregoing equations $U_{i}$ designates the velocity and $v$ stands for the kinematic viscosity of the fluid. The vorticity transport equation is written for the total quantities, i.e., the mean plus the fluctuating values of vorticity and velocity. stretching and tilting are embodied in the vorticityvelocity gradient interaction term $\omega_{j} \partial U_{j} / \partial x_{j} \cdot$ This nonlinear term is encountered only in three dimensional flows. Turbulence, as a matter of fact, is always three dimensional even when the mean flow is one or two dimensional. stretching (or squeezing) of vortex filaments along their axes without affecting their directions is outlined by the three terms $\omega_{j} \partial U_{i} / \partial x_{j}$ when $i=j$. Tilting of the vortex filaments is represented by the six terms $\omega_{j} \partial U_{i} / \partial x_{j}$ when $i \neq j$.

For the sake of illustrating the stretching mechanism and the tilting effect, cross flow about an infinitely long circular cylinder neglecting viscous dissipation is examined. The physical situation is thus that of a diverging stagnation-point flow around a circular cylinder of radius $R$. It is portrayed along with the system of coordinates used in Fig. 1. It is further assumed that the approaching velocity $\mathrm{U}_{2}$ contains mainly cross vorticity susceptible to undergoing stretching in the diverging stagnationpoint flow. In other words, only that component of vorticity whose axis is aligned with the $x_{1}$-direction, $i . e$. the $\omega_{1}$-component, is accounted for. The other two components are disregarded in order to focus on the stretching and the tilting of cross-vortex tubes.

To inspect these two mechanisms, a single ideal cross-vortex tube of a scale $\lambda$ larger than the neutral scale $\lambda_{0}$ is considered in the $x_{1} x_{2}$-plane (or the stream plane) as depicted in 
Fig. 1. As this cross-vortex tube is swept by the mean flow toward the stagnation zone it undergoes axial stretching in the $\mathrm{x}_{1}$-direction due to the favorable rate of tensile strain, i.e., due to the prevailing positive velocity gradient in the $x_{1}-$ direction $\partial U_{1} / \partial x_{1}$. This suitable tensile strain rate is induced by the divergence of the streamlines in the stagnation flow. Throughout the stretching both the volume and the angular momentum of each cross-vortex filament are conserved as a result of discarding the viscous dissipation. Elongation of a cross-vortex tube is consequently accompanied by a decrease in its scale (or diameter) $\lambda$ and by an increase in its vorticity (or angular velocity) $\omega_{1}$. Then the turbulent velocity $u_{2}$ in the plane normal to the axis of the cross-vortex tube is amplified. Turbulent kinetic energy consequently concentrates within the stretched cross-vortex filaments. Illustration of the axial stretching of an ideal cross-vortex tube initially at some upstream station $x_{2}^{\circ}$ is provided in Fig. 1. As this cross-vortex tube approaches the stagnation zone, its axial elongation and concurrent scale diminution along with the accompanying amplification of both crossvorticity $\omega_{1}$ and streamwise turbulence $u_{2}$ are portrayed at two streamwise stations $x_{2}^{\prime}$ and $x_{2}^{\prime \prime} \cdot$ Stretching is thus an ordered mechanism that occurs in preferred directions according to the prevalent tensile strain rates. In a general three-dimensional flow of an incompressible fluid, stretching can take place, at most, in two directions. In the third direction the vortex tubes experience squeezing due to the constraints imposed on the rates of tensile strain by the continuity equation. One can view the cross-vortex tubes being conveyed by the diverging stagnation flow as an organized substructure subsisting within the overall random turbulent field. The stretching represents the triggering agent needed to unfold this latent coherent substructure to its fullest manifestation.

Concurrently with the stretching, each cross-vortex tube undergoes tilting as it is nearing the body. The tilting is caused by the cross gradient of the approaching velocity $\mathrm{U}_{2}$, i.e., by $\partial U_{2} / \partial x_{1}$. This velocity cross gradient is, in turn, produced by the diverging stagnation flow. A cross-vortex filament $\omega_{1}$ in the $x_{1}$-direction acquires as a result of its tilting a vorticity component $\omega_{2}$ in the $x_{2}$-direction and, therefore, its vorticity becomes $\vec{w}_{1}+\vec{w}_{2}$. The tilting of an ideal cross-vortex tube initially with its axis in the $x_{1}$-direction at some station $\mathrm{x}_{2}$ is portrayed in Fig. 2 at two streamwise stations $x_{2}^{\prime}$ and $x_{2}^{\prime \prime}$ in the stream plane (the $x_{1} x_{2}$-plane). As the body is approached and the cross-vortex filaments are conveyed past the body, the tilting effect becomes more pronounced. This increase in tilting is shown by the angle $\theta$ in Fig. 2. Essentially, the tilting is responsible for a continuous back-and-forth exchange of vorticity (or turbulent kinetic energy) among the components of turbulent vorticity. In the case of stagnation-point flow the tilting of the stretched vortex tubes is distinctly biased in the streamwise direction. The tilting consequently acts in an 
organized manner in this flow. An overall picture of both stretching and tilting of a cross-vortex tube at three streamwise stations $x_{2}^{\prime}, x_{2}^{\prime \prime}$ and $x_{2}^{\prime \prime \prime}$ as it is nearing a circular cylinder is provided in Fig. 3. Initially, this cross-vortex tube was oriented in the $x_{1}$-direction at station $x_{2}^{\circ}$. The three planes of the flow-viz., the stream, the normal and the cross planes-are further delineated in Fig. 3.

The stretching and the accompanying tilting lead to the development of an organized cellular flow pattern near the stagnation zone of the body. Ideally, this coherent substructure consists of a regular array of standing cross-vortex tubes with their axes parallel to the streamlines along the body and with their cores outside the boundary layer. Characteristic to this array is the orderly spanwise distribution of repetitive cells of equal scales within which the rotation alternates in its direction. Turbulent energy accumulates within these turbulent cross-vortices owing to the stretching. Most of the turbulence amplification occurs at some particular scale larger than the neutral one, i.e., at a most amplified scale $\lambda_{\mathrm{m}}$ (ref. 7 ). This most amplified scale $\lambda_{m}$ is generally greater than but commensurate with the body boundary-layer thickness $\delta$. A schematic of the coherent vortex flow pattern near the stagnation zone in the $x_{2} x_{3}-p l a n e$ (the normal plane) is displayed in Fig. 4. The most amplified scale $\lambda_{\mathrm{m}}$ is indicated in this figure. In addition, the theoretical boundary layer $\delta$ (ref. 6) is marked in this figure by a dashed line. Discrete vortices (or eddies) are continuously drawn out from the stagnation-zone array of standing vortexes and, subsequently, swept downstream by the mainflow round the body. As a result, they possess the capability to penetrate into the prevailing boundary layer. Their interaction with the boundary layer is feasible provided that their scale is commensurate with the boundary-layer thickness. This is exactly the situation considering that the most amplified scale $\lambda_{m}$ is of the same order of magnitude as the boundary-layer thickness (ref. 7). The eddies penetrating the boundary layer affect its properties through energizing it. A supply of adequate amplified turbulence can lead to arresting the growth or even to the forestalling of laminar separation. Realization of the latter is contingent upon shifting the transition to a turbulent boundary layer at the stagnation point of the body.

Visual observations, since the first experiments of Reynolds (ref. 13), represent an indispensable diagnostic tool in turbulent flow research. Flow visualization possesses the potential to furnish invaluable physical insights into the patterns of turbulent flows despite its inherent limitations. Its main salient trait is the ability to render a Lagrangian description of the flow. The usefulness of optical techniques regarding identification of spatially coherent patterns of turbulence have particularly been demonstrated. Recent visualization studies have revealed that turbulence is less chaotic than generally assumed in many 
particular flow situations (refs. $7,14,15,16$; to mention a few). In carrying out flow visualization it is of utmost importance to pay adequate attention to its limitations which stem from the instantaneous interpretation and the two-dimensionality of the pictures. These intrinsic restrictions are however overridden by the significant physical evidence supplied by the visualization and its prompting of a better understanding of the coherent structure of the flow. The visualization moreover possesses the capability to furnish reasonable quantitative data concerning the coarse flow structure.

An extensive, albeit not exhaustive, visualization of the flow near the stagnation zone of a circular cylinder is reported herein. The primary motivation of this visualization study was to outline the selective stretching mechanism and the tilting of cross-vortex tubes. An attempt at delineating the coherent vortex structure near the stagnation zone was concurrently carried out. An additional objective was to identify the interaction between the amplified turbulence and the body laminar boundary layer. Quantification of the flow images was furthermore sought for estimating the time evolution of the flow pattern and its significant scales. 
The flow vi.sualization was conducted in a low-speed closedcircuit wind tunnel at the Fluid Dynamics and Diffusion Laboratory, Colorado state University. This wind tunnel has a cross section of $1.83 \times 1.83 \mathrm{~m} \mathrm{(6 \times 6} \mathrm{ft),} \mathrm{a} \mathrm{test} \mathrm{section} 27 \mathrm{~m} \mathrm{(88} \mathrm{ft)} \mathrm{long} \mathrm{and} \mathrm{a}$ contraction ratio of $9: 1$. To facilitate flow visualization, the side parels and parts of the ceiling of the test section are made of high quality tempered glass and Plexiglas. Throughout the flow visualization the freestream velocity $U_{2}$ was maintained at 0.76 $\mathrm{m} / \mathrm{s}(2.5 \mathrm{ft} / \mathrm{s})$ and the turbulence intensity immediately upstream of the turbulence-generating grid was about $1.0 \%$. The freestream velocity was controlled at this low range within a resolution of roughly $35 \%$.

A circular cylinder $16 \mathrm{~cm}(6-1 / 4 \mathrm{in})$ in diameter $\mathrm{D}$ and 1.83 $\mathrm{m}$ (6 ft) long made of light aluminum alloy was used. The cylinder was mounted across the width of the wind tunnel at $23 \mathrm{~m}$ ( 75 ft) downstream of the test-section entrance with its axis $0.61 \mathrm{~m}$ ( 2 ft) above the floor. The blockage coefficient was about 0.087. To provide a satisfactory background and contrast for the visualization, the cylinder surface was painted with dead black lacquer. Additionally, the wind-tunnel floor was painted with flat black paint. A schematic diagram of the experimental arrangement (top view, i.e., in the $x_{2} x_{3}$-plane) including all important dimensions and the system of coordinates used is displayed in Fig. 5 .

The visualization was carried out at a Reynolds number of about $8 \times 10^{3}$. This Reynolds number $\operatorname{Re}_{D}$ was based on the freestream velocity $\left(U_{2 \infty}=0.76 \mathrm{~m} / \mathrm{s}\right)$ and the cylinder diameter $(D=.6$ $\mathrm{cm}$ ) in air at $20^{\circ} \mathrm{C}\left(68^{\circ} \mathrm{F}\right.$ ) (kinematic viscosity $\nu=1.5 \times 10^{-5}$ $\left.\mathrm{m}^{2} / \mathrm{s}\left(1.6 \times 10^{-4} \mathrm{ft}^{2} / \mathrm{s}\right)\right)$. At this Reynolds number a neutral wavelength of $5.6 \mathrm{~mm}(0.22 \mathrm{in})$ and a stagnation laminar boundary layer $2.15 \mathrm{~mm}(0.08 \mathrm{in})$ thick are expected. The neutral scale $\lambda_{0}$ and the laminar boundary-layer thickness $\delta$ were computed by means of the relationships (ref. 6)

and

$$
\lambda_{O}=2 \pi R / R e_{D}^{1 / 2},
$$

$$
\delta=2 \cdot 4 R / R e_{D}^{1 / 2}
$$

where $R$ is the cylinder radius $(8 \mathrm{~cm}(3-1 / 8$ in)).

Production of turbulence (or vorticity) in a controlled manner upstream of the cylinder was achieved utilizing a turbulencegenerating grid. This turbulence-generating grid consisted of 24 regularly spaced vertical cylindrical bars that spanned the entire height of the wind-tunnel cross section $(1.83 \mathrm{~m})$. Aluminum rods, each $1.27 \mathrm{~cm}(1 / 2 \mathrm{in})$ in diameter, spaced at center-to-center intervals of $6.35 \mathrm{~cm}(2-1 / 2 \mathrm{in})$ were employed. Thus, the mesh or 
the gap between two rods was $5 \mathrm{~cm}$ (2 in) which in terms of the cylinder diameter $(16 \mathrm{~cm})$ was $0.32 \mathrm{D}$. For this mesh the corresponding Reynolds number (based on the freestream velocity) was about 2600. The grid was mounted $116 \mathrm{~cm}(45-3 / 4 \mathrm{in})$ upwind of the cylinder as shown schematically in Fig. 5. In terms of the cylinder diameter $D$ or the rod diameter $d$, this upstream distance was 7.32D or 91.5d. Grid-generated turbulence decays quite rapidly with the downstream distance in the absence of a downstream body. It becomes practically isotropic within a downstream distance of 30 to at most 60 rod diameters depending upon the grid geometry and the grid Reynolds number based on the mesh (refs. 7 and 17).

The vertical orientation of the rods was selected for the sake of governing the generation of upstream turbulence (or vorticity) in the way required for the specific stagnation-flow situation. Insofar as the cylinder was mounted horizontally, the goal was to produce mainly vorticity oriented in the $x_{1}$-direction as depicted in Figs. 1 and 3. This is that particular component of a general three-dimensional vorticity field susceptible to undergoing amplification by stretching in the stagnation-flow considered. Furthermore, this vorticity component is also subjected to the appropriate tilting portrayed in Fig. 2 . It is important to stress that the turbulence-generating grid was conceived specifically for producing chiefly a vorticity component in the $x_{1}$-direction at scales larger than the neutral ones.

The Reynolds number of each individual rod of the grid was about 650. It was based on the freestream velocity $U_{2 \infty}$ and the rod diameter $(d=1.27 \mathrm{~cm})$ in air at $20^{\circ} \mathrm{C}$. The corresponding Strouhal number $\mathrm{S}-\mathrm{i} . \mathrm{e}, \mathrm{S}=\mathrm{nd} / \mathrm{U}_{2 \infty}$, where $\mathrm{n}$ is the frequencywas roughly 0.21 (ref. 18). Most of the turbulent energy was consequently concentrated at a strouhal frequency of about 12.6 $\mathrm{Hz}$. As a result, the strouhal scale $\lambda_{\mathrm{S}}=\mathrm{U}_{2 \infty} / \mathrm{n}$, approximated under the assumption of a frozen pattern (ref. 19), was $60.45 \mathrm{~mm}$ (2.38 in). Turbulence was thus mainly produced at a scale almost 11 times greater than the neutral scale of the stagnation flow $\left(\lambda_{\mathrm{o}}=5.6 \mathrm{~mm}\right)$. The turbulence-generating grid therefore met the necessary criteria for producing the wanted turbulence. 
The freestream velocity was monitored by means of a Pitotstatic tube located $10.36 \mathrm{~m}$ (34 ft) upwind of the cylinder in the plane $x_{3}=0$ ( $i . e .$, in the $x_{1} x_{2}$-plane or the stream plane) as indicated in Fig. 5. A Pitot-static tube of modified Prandtl type $3.2 \mathrm{~mm}(1 / 8 \mathrm{in})$ in diameter with an impact-pressure orifice 0.79 $\mathrm{mm}(1 / 32 \mathrm{in})$ in diameter was utilized (Pitot-static tube, Model PAC-12-KL, United Sensor \& Control Corp., Watertown, MA). The Reynolds number of this tube, based on its outside radius $(1.59 \mathrm{~mm}$ $(1 / 16 \mathrm{in})$ ) at the low freestream velocity used $(0.76 \mathrm{~m} / \mathrm{s})$, was about 80. Fortunately, no viscous correction is necessary when this Reynolds number is larger than 10 (ref. 20). The reading of the Pitot-static tube was not affected by the superimposed turbulence since it was positioned upstream of the turbulence-generating grid. Within this region of the test section the effect of the wind-tunnel background turbulence intensity, which was of the order of 0.1\%, is completely negligible (refs. 21 and 22). An electronic pressure meter (MKS Baratron Type 77 with Type $77 \mathrm{H}$ Series Pressure Heads, MKS Instruments, Inc., Burlington, MA) with a resolution of $0.0005 \mathrm{~mm} \mathrm{Hg}$ was utilized to measure the dynamic pressure. The stream temperature was monitored continuously by means of a mercury thermometer with a resolution of $0.1^{\circ} \mathrm{C}\left(0.18^{\circ} \mathrm{F}\right)$ (Brooklyn Thermometer Co., Farmingdale, NY). It was located in the same plane as the Pitot-static tube as shown in Fig. 5 .

The flow visualization was carried out utilizing white smoke composed of titanium dioxide $\left(\mathrm{TiO}_{2}\right)$. This white smoke was produced by mixing moist compressed air with titanium tetrachloride $\left(\mathrm{TiCl}_{4}\right)$ according to the reaction $\mathrm{TiCl}_{4}+2 \mathrm{H}_{2} \mathrm{O} \rightarrow \mathrm{TiO}_{2}+4 \mathrm{HCl}$. One and/or two smoke generators (CSU Smoke Generator) were employed to produce the $\mathrm{TiO}_{2}$ at the desired flow rates. Smoke was injected through a single and/or two brass tubes $3.2 \mathrm{~mm}(1 / 8$ in) in diameter (O.D.) connected through Tygon tubes to the smoke generators. Selection of this diameter ensured the total neglect of the tube wake effect upon the smoke flow. All the tubes were positioned 5 $\mathrm{cm}$ (2 in) upstream of the cylinder in the plane $x_{1}=0$ (i.e., in the $x_{2} x_{3}-p l a n e$ or the normal plane) at angles of either $0^{\circ}$ or $30^{\circ}$ with respect to the $\mathrm{x}_{2}$-axis. In terms of the cylinder diameter $(16 \mathrm{~cm})$ this distance was 0.32D. Each smoke injection tube was fastened to a fine stand that was located near the wind-tunnel side wall. Extreme care was taken to match the smoke exit velocity to the prevalent stream velocity for ensuring its neutral entrainment. A sketch of the smoke injection arrangement is provided in Fig. 6 .

Still photographs were taken using a motor-driven single-lens reflex Hasselblad camera (Model 500 EL/M). A Carl Zeiss planar standard lens with an $80 \mathrm{~mm}$ focal length and an f-stop of $f / 2.8$ was utilized. This lens was selected after an exhaustive sequence 
of trials. Black-and-white stills were made employing Kodak Tri-X Pan and/or Plus-X Pan films. The former is a Tri-X 120 high-speed panchromatic film (ASA 400 (27 DIN)) with fine grain. It is especially suited for photographing fast action of subjects in relatively dim light that requires good depth-of-field and a high shutter speed (ref. 23, pp. DS 24-25). Standard-size film rolls of 16 exposures were employed. The Kodak Plus-X Pan film is a medium-speed panchromatic film (ASA 125 (22 DIN)) with very fine grain, high resolving power and excellent sharpness (ref. 23, pp. DS 12-13). In the base material of this film a gray dye is included for enhancing antihalation and for preventing edge fog. Standard cassettes of this Plus-X $488 \mathrm{film}$ in $4.6 \mathrm{~m}$ ( $15 \mathrm{ft}$ ) long rolls consisting of 70 exposures per roll were used. The picture size of both Tri-X and Plus-X films is $6.35 \mathrm{~cm}$ square $(2-1 / 2$ in square). All the black-and-white still photographs were snapped at an aperture ranging from $f / 2.8$ to $f / 22$ and shutter speeds within the range $1 / 60$ to 1 s. In addition, a Polaroid Land camera (Polaroid, Model 180, Polaroid Corp., Cambridge, MA) equipped with a Tominon lens with a focal length of $114 \mathrm{~mm}$ and an f-stop of f/4.5 was utilized for supplying immediate prints. Polaroid Type 667 coaterless black-and-white Land pack films were employed. This is a panchromatic (type B) very high speed film (ASA 3000 DIN)) with print size $8.3 \times 10.8 \mathrm{~cm}(3-1 / 4 \times 4-1 / 4 \mathrm{in})$.

Black-and-white $16 \mathrm{~mm}$ movies were taken using a cine-Kodak Special camera (Eastman Kodak Co., Rochester, NY) equipped with a Kodak anastigmatic lens with a focal length of $63 \mathrm{~mm}$ and an f-stop of f/2.7. Kodak black-and-white Tri-X reversal film 7827 (ref. 24) was utilized. This is a high-speed panchromatic film with exposure indexes of ASA 200 (24 DIN) in daylight and ASA 160 (23 DIN) for Tungsten lighting $(3200 \mathrm{~K})$. The Tri-X film possesses excellent tonal gradation and halation control. This film is particularly useful under indoor difficult lighting conditions. Color $16 \mathrm{~mm}$ movies were made employing a Bolex H-l6 SBM reflex camera equipped with a built-in governor-controlled spring motor (Paillard, S. A., Sainte-Croix, Switzerland). This camera allows through-the-lens viewing during filming and when it is not running. Its reflex viewfinder permits accurate framing and focusing, estimation of the depth-of-field, determination of the correct focal length and evaluation of the desired illumination. A power zoom lens with automatic through-the-lens exposure control (Vario Switar 100 POE, Kern-Paillard, Bolex Internationa1, S.A., SainteCroix, Switzerland) was used in conjunction with the Bolex H-16 camera. This is a versatile power zoom lens with a focal length and an aperture ranging from 16 to $100 \mathrm{~mm}$ and from $\mathrm{f} / 60$ to f/I.9,

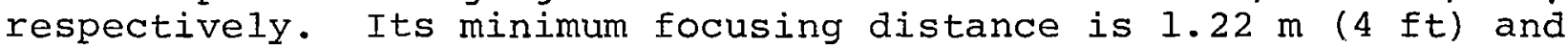
the corresponding smallest area covered is $8.6 \times 6.7 \mathrm{~cm}(3-3 / 8 \times 2-5 / 8$ in). High-speed color-reversal films (Kodak Ektachrome EF 7242 (Kodak 1974)) with exposure indexes of ASA 80 (20 DIN) in daylight and ASA 125 (22 DIN) for Tungsten lighting $(3200 \mathrm{~K})$ were employed. This Kodak film is specially designed for indoor use under lowlevel lighting conditions. Both the black-and-white and the color 
motion pictures were shot at a speed of 24 FPS (frames per second) and, therefore, the time interval of each individual frame was 41$2 / 3 \mathrm{~ms}$.

Lighting was supplied by means of floodlights and spotlights (Berkey Photo Inc., Burbank, CA). One fixed focus floodlight (Colortran $650 \mathrm{~W} \mathrm{Mini}-6$ ) and three lamps with variable focus from floodlight to spotlight (two Colortran $650 \mathrm{~W}$ Multi-6 and one Colortran 1,000 $\mathrm{W}$ Multi-10) were employed. Iight sheets were produced, whenever necessary, using the built-in barn doors. Adjustment of the light intensity was controlled using variacs (10 A Type W-10, General Radio Co., Concord, MA; Model 7.5 A, Superior Electric Co., Bristol, CN). Both the stills and the motion pictures were taken from three main view angles, viz., side, top and rear views. In each photographic situation the composition of the lamps and their light intensity were determined for illuminating the smoke and minimizing the glare induced by the smooth surface of the cylinder. The main light source (a Multi-6) and the second or fill-in lamp (a Multi-6) were placed upwind of the cylinder. An accent light (a Multi-10), i.e., the third lamp, was placed behind the cylinder. The fourth light source or the background light (a Mini-6) was located underneath the cylinder. A typical lighting composition for the top-view photography is shown in Fig. 7. A reflected/incident-light meter (Luna-Pro Electronic System Exposure Meter, Gossen GmBh, Erlangen, West Germany) was utilized for determining the accurate exposure for each particular lighting setup. 


\section{EXAMINATION OF FLOW EVENTS}

The main objectives of the flow visualization study were: (1) demonstration of the stretching of cross-vortex tubes and of its effects; (2) illustration of the tilting of cross-vortex tubes; (3) delineation of the coherent vortex structure near the stagnation zone of the cylinder; (4) identification of the interaction between the amplified turbulence and the cylinder laminar boundary layer; and, (5) outlining the development of a turbulent boundary layer. In order to achieve these aims the smoke patterns were photographed, as previously mentioned, from three viewpoints, viz., side, top and rear views. The flow events were consequently visualized in the planes $\mathrm{x}_{1} \mathrm{x}_{2}, \mathrm{x}_{2} \mathrm{x}_{3}$ and $\mathrm{x}_{1} \mathrm{x}_{3}-\mathrm{i} . \mathrm{e}$, , in the stream, normal and cross planes (see Fig. 3). This threefold visualization permitted distinct perception of all the relevant features of the flow.

An overall examination of more than a hundred still photographs and more than $610 \mathrm{~m}(2000 \mathrm{ft})$ of $16 \mathrm{~mm}$ movies was carried out. They were analyzed in various degrees of detail depending upon their particular view angles. By and large, the analysis of the flow events was a painstaking effort and an extremely time consuming task owing to the complexity of the flow. This endeavor was, nevertheless, highly rewarding since the flow pictures supplied invaluable physical insights into the intricacies of the flow. The still photographs revealed numerous significant aspects of the flow pattern. However, they rendered solely overall qualitative synoptic views because of their static nature. The motion pictures, on the other hand, furnished a comprehensive understanding of the time-space development of the stretching and its related effects. As a matter of fact, the motion pictures were instrumental in achieving the set goals of the visualization study. Static presentation of stills from movie strips, as discussed herein, despite its worthy return cannot match the dynamic impact conveyed by the motion pictures. A summary movie entitled "Vorticity Amplification in Stagnation Flow about a Circular Cylinder" that incorporates the most instructive views was consequently edited and is available for loan*.

The task at hand was to interpret both qualitatively and quartitatively the motion pictures for the sake of ascertaining the development in time and space of the flow pattern. Such interpretations are highly tempting in view of the Lagrangian description supplied by them. Continuous sequences of individual frames from numerous movie strips were analyzed for each particular view angle. Extreme care was exercised in analyzing the motion pictures owing to the associated inherent restrictions previously mentioned and allowance was made for the inevitable

* See description on card at back of report. 
optical distortions. Each single frame, whose significant size was about $10 \times 7.5 \mathrm{~mm}$ (width $x$ height), was magnified 61 times by projecting it onto a $61 \times 45.75 \mathrm{~cm}(24 \times 18$ in) white drawing paper. Inspection of each frame was accomplished with the aid of a special $16 \mathrm{~mm}$ photo optical data analyzer movie projector ( $\mathrm{L}-\mathrm{W}$ Photo Data Analyzer Movie Projector, Model 224-A, L-W Photo, Inc., Van Nuys, CA). This movie projector can be used at normal speeds of 16 or 24 FPS or as a stop motion analytical projector. In the latter mode projection at $1,2,4,6,8$ and/or 12 FPS is available. Moreover, this movie analyzer permitted a complete stopping of the action indefinitely at any desired frame for close examination. In carrying out the projection a wide angle and high speed lens with an effective focal length of $15.875 \mathrm{~mm}$ (5/8 in) and an f-stop of 1.4 (Model 663-430, Buhl Optical Co., Pittsburg, PA) was utilized.

The pattern of the smoke filaments entrained by a cross-vortex tube was tracked for each single frame on its magnified image. In drawing the pathlines (or the contour lines) the distortions resulting from the inevitable two-dimensional projection were accounted for by comparing images from different view angles. Every necessary effort for achieving the best possible accuracy in consummating the tracing of the flow pattern was undertaken. In some instances, however, attainment of all the details was hindered by the complexity of the patterns. Whenever these situations were met only the significant features of the flow patterns were retained in tracing the schematics. The frame by frame scrutiny of the movie strips led, despite its limitations, to the acquisition of a reasonably quantitative interpretation of the gross flow structure in addition to supplying an in-depth qualitative apperception of the flow pattern.

Interpretative sketches of sequences up to 20 frames, at most, were edited for each particular view angle. As a result, the flow evolution in time was followed over time intervals up to 833-1/3 ms, as the time exposure of every frame was $41-2 / 3 \mathrm{~ms}$ (24 FPS). Stills of selected movie-strip frames along with their corresponding schematics of the pathlines are displayed hereinafter. All the stills given are enlarged by only 6 times in the light of the space constraints and, therefore, their dimensions are $60 \times 45 \mathrm{~mm}(2-3 / 8 \times 1-13 / 16 \mathrm{in})$. For the sake of consistency, the derived sketches presented are of about the same size as their still counterparts. They were obtained by reducing the original schematics to about $10 \%$ of their initial size. In analyzing the sketches of the smoke pattern a length scale was estimated in each case based on the known upstream distance of the smoke injection tube which was located $5 \mathrm{~cm}$ upwind of the cylinder (see sect. 3). The scale, the time axis (t-axis) and the time interval of each frame, the frame counter $N$, the system of coordinates and the total approaching velocity are shown in all the figures. In addition, the theoretical laminar boundary-layer thickness, which was $2.15 \mathrm{~mm}$ at the stagnation zone (see sect. 2), is indicated on 
all the sketches by a dashed line and the letter $L$. The cylinder itself is marked off in the schematics by a solid line. As the analysis of the flow pictures is reported, pertinent discussions are interspersed wherever they are deemed helpful for the proper interpretation of the images. In carrying out the analysis of the flow motion pictures, the effects of viscous dissipation were neglected since the primary objective was to demonstrate the stretching mechanism and its consequences.

\subsection{Side Views}

Remarkable overall displays of the flow structure were revealed by the side views in the $x_{1} x_{2}-p l a n e$ or the stream plane (see Fig. 3). An instructive illustration of the information embedded in these views is rendered by the still photograph and the accompanying blowup ( $x 5$ enlargement) of the foreground image near the stagnation zone given in Fig. 8. This still was taken at an aperture of $f / 2.8$ and at a shutter speed of $1 / 60 \mathrm{~s}$. A sketch showing the smoke injection is incorporated in this figure. To start with, it is important to notice the smoothness of the smoke filament ejecting from the tube. This clearly indicates the matching of the smoke exit velocity with the mainstream velocity and the lack of any significant wake effect of the smoke tube. The entrainment of the smoke filament by the vortex emerging near the stagnation zone is distinctly observed in the two photographs. It is further evident that the axis of this vortex tube is aligned with the $x_{1}$-direction, i.e., a cross-vortex tube. Occurrence of stretching action is inferable from the distinctly spiral shape of the cross-vortex tube in the foreground of the photograph which is particularly noticeable in the enlarged print. The tilting of the cross-vortex tube is indicated by the sweeping of the smoke above the cylinder. Finally, the spiraling path above the body is suggestive of a prevailing turbulent boundary layer.

The time history of a cross-vortex tube since its "embryo" until its "aging" was best perceived in the side-view motion pictures. A sequence of 18 stills from a representative movie strip extending over a time span of $750 \mathrm{~ms}$ along with their corresponding schematics is shown in Figs. 9.1 to 9.5. Note that the mirror image of the smoke pattern is reflected on the cylinder surface. In these figures $S$ designates side view, and the scale of the sketches is $1: 3(1 \mathrm{~mm}=3 \mathrm{~mm})$. Additionally, the thickening of the theoretical laminar boundary layer along the cylinder circumference from $2.15 \mathrm{~mm}(0.08 \mathrm{in})$ at the stagnation zone up to about $6 \mathrm{~mm}(0.24 \mathrm{in})$ at an angle of 80 to $90^{\circ}$ (measured from the stagnation point) is shown in all the sketches (ref. 25). In analyzing. the movie strip the goal was to track the sequence of events or the "life cycle" of a cross-vortex tube since its onset near the stagnation zone throughout its penetration and blending into the boundary layer. Consequently, in tracing out the sketches the vestiges of smoke entrained by the previous cross-vortex tube 
were disregarded although they are clearly observed in the stills. The initial and the subsequent gradual entrainment of the approaching smoke into a newly arrived cross-vortex tube near the stagnation zone is noticed in the first four frames, viz., in frames $1-S$ to $4-S$, given in Fig. 9.1. One can view the corresponding time interval up to about $t=166-2 / 3 \mathrm{~ms}$ (frame $4-\mathrm{s}$ in Fig. 9.1) as indicative of the nascent phase as regards the emerging of the cross-vortex tube near the stagnation zone. During this embryonic stage the stretching was not yet fully discernible.

The maturing process of the cross-vortex tube becomes prominent starting from $t=208-1 / 3 \mathrm{~ms}$ (frame 5-S in Fig. 9.2). Axial stretching in the $x_{1}$-direction is seen coming into play from $t=$ $208-1 / 3$ to $375 \mathrm{~ms}$ (images $5-\mathrm{S}$ to $9-\mathrm{S}$ shown in Figs. 9.2 and 9.3). As a cross-vortex tube is stretched, its angular velocity increases owing to the conservation of angular momentum. This augmentation in the angular velocity promotes enhanced entrainment of the smoke within the cross-vortex tube. The net result is a clearly outlined stretched cross-vortex tube as observed in images 5-s to 9-S and in the corresponding schematics (Figs. 9.2 and 9.3). In scrutinizing the shape of the cross-vortex tube delineated in the schematics it is apparent that its core is outside the laminar boundary layer.

Ascertainment of the spatial scales of the cross-vortex tubes in the stream plane was next sought from the interpretative sketches. These scales were simply approximated by measuring the width of the tubes traced on the schematics according to the scale of 1:3. They ranged roughly from 4 to $40 \mathrm{~mm}(0.16$ to $1.60 \mathrm{in})$, as indicated in the schematics of the pathlines. The largest scale of the tube or its envelope is marked on the sketches by a broken line (--). All these scales are gross estimates considering the intrinsic approximations associated with the tracing of the flow patterns. However, they are undoubtedly representative of the order of magnitude of the large scales of the stretched crossvortex tubes. In accordance with the vorticity-amplification theory (see sect. 2, Eq. (3)) the neutral scale for this flow $\lambda_{O}=$ $5.6 \mathrm{~mm}$. Therefore, vorticity at scales larger than the neutral one prevails near the stagnation zone. This is exactly the cross vorticity that undergoes amplification by stretching. In all likelihood the scales smaller than the neutral wavelength delineate cross-vortex. tubes that already underwent stretching. The occurrence of selective stretching of cross-vortex tubes, as depicted in Fig. 1, is thus substantiated by the observed flow pattern. This stretching leads to the amplification of the cross vorticity $\omega_{1}$ and, consequently, to the amplification of the streamwise turbulent velocity $\mathrm{u}_{2}$.

Tilting in the streamwise direction of the tracked crossvortex tube is noticed during its maturing phase in the stills shown in Figs. 9.2 to 9.4. In tracing the tilting development 
attention was paid primarily to the gross scale in view of the observed severely distorted flow structure. Nevertheless, the significant patterns of the deformed flow were traced out. With the beginning of the mature phase the tilting is discerned. In still 6-S shown in Fig. 9.2 (250 ms), the winding of the smoke filament over the cylinder indicates the start up of the vortextube tilting. The wrapping of the stretched cross-vortex tube around the cylinder indicates how the tilting grows. As the stretched tube moves downstream, the tilting becomes, as expected, more pronounced with increasing time. The tilting is furthermore accompanied by a continuous expansion of the cross-vortex tube. For instance, the gross scale expands from about $18 \mathrm{~mm}(0.71$ in) at $t=250 \mathrm{~ms}$ (frame $6-\mathrm{S}$ in Fig. 9.2) to roughly $40 \mathrm{~mm}$ (1.60 in) at $t=666-2 / 3 \mathrm{~ms}$ (frame 16-S in Fig. 9.4). Recall that this gross scale is annotated on the sketches by a broken line. The systematic biasing of the tilting in the streamwise direction, as portrayed in Fig. 2 , is unveiled by the visualized blow pattern.

In the life cycle of the tracked vortex tube the penetration of the amplified vorticity (or amplified turbulence) into the laminar boundary layer at the stagnation zone is apperceived beginning from about $t=125$ to $166-2 / 3 \mathrm{~ms}$ (frames $3-S$ to $4-S$ in Fig. 9.1). It becomes more forceful and deeper with increasing time as distinctly observed in the subsequent images shown in Figs. 9.2, 9.3 and 9.4. The encroaching inroads into the boundary layer along the cylinder are fully manifested starting from about $t=333-1 / 3$ to $375 \mathrm{~ms}$ (frames $8-\mathrm{s}$ and 9-S in Figs. 9.2 and 9.3). As the interaction with the boundary layer progresses, the distortion of the flow pattern within a continuously distending vortex tube becomes more conspicuous. The agitated state of the smoke filaments unraveled by the prevailing spiral pattern is the prominent feature of this distorted flow. In the sketches of the frames $13-S$ to $16-\mathrm{S}$ ( $t=541-2 / 3$ to $666-2 / 3 \mathrm{~ms}$ in the life cycle) given in Fig. 9.4, this spiral pattern is particularly discriminated. This overall disordered pattern of the smoke filaments attests to the development of a turbulent boundary layer. It is thus apparent that the stretching of the cross-vortex tubes and their streamwise tilting lead, in all likelihood, to the development of a turbulent boundary layer.

The tracked vortex tube is gradually losing its identity with its maturing. Its growth within the time interval from $t=166-$ $2 / 3$ to $666-2 / 3 \mathrm{~ms}$ (frames $5-\mathrm{s}$ to $16-\mathrm{s}$ in Figs. 9.2 to 9.4) is characterized by an incessant evolvement into a chaotic flow pattern which is effected by the development of the turbulent boundary layer. As the vortex tube reaches its aging phase another cross-vortex pattern is seen to show up near the stagnation zone and enter upon a similar sequence of events. During this aging stage the remnants of the tube are pulled over and beneath the cylinder. Essentially, these remnants are equivalent to the smoke vestiges of the previous tube which were initially disregarded in tracing the evolution of this particular tracked 
cross-vortex tube. The aging of the tracked tube and the onset anew of a cross-vortex tube near the stagnation zone are readily identified starting from $t=708-1 / 3 \mathrm{~ms}$ in frames $17-\mathrm{s}$ and $18-\mathrm{s}$ displayed in Fig. 9.5. It is important to notice that the large scale of the newly emerging cross-vortex tube is roughly of the same size as that of the aging one at a corresponding stage in its growth. The embryonic phase of this new tube is noticeable in frames 13-S to I6-S shown in Fig. 9.4. Basically, the foreground images in these four frames, which extend over a time interval of 166-2/3 $\mathrm{ms}$, strongly resemble those of the older tube displayed in frames $1-S$ to $4-S$ in Fig. 9.1. A gross scale of about $15 \mathrm{~mm}(0.60$ in) was further estimated for the new tube as indicated in sketches 17-S and 18-S (Fig. 9.5). The older tube exhibited roughly the same size coarse scale after about $250 \mathrm{~ms}$ in its life cycle as depicted in Fig. 9.2. In practice, frame 18-S is at $t=$ $250 \mathrm{~ms}$ with regard to the new tube. It is thus apparent that the life cycle of a vortex tube from its embryonic through its aging phases extends over a time interval of about 700 to $750 \mathrm{~ms}$ at the particular Reynolds number $\left(8 \times 10^{3}\right)$ for this flow situation. Analysis of other side-view sequences confirmed this time span within \pm 5 to $10 \%$.

\section{2 Top Views}

Top views taken from directly above the stagnation zone clearly exhibited the occurrence of an organized vortex flow pattern in the $\mathrm{x}_{2} \mathrm{x}_{3}-\mathrm{plane}$ which is the normal plane due to its orientation (see Fig. 3). A comprehensive insight into the information contained within the top views in the normal plane is supplied by the snapshot and the enlargement (10X magnification) of its foreground image given in Fig. 10. This still photograph was taken at an $\mathrm{f} / 4.5$ aperture and a shutter speed of $1 / 60 \mathrm{~s}$. A schematic of the smoke injection configuration is included in this figure. The circular shape of the smoke engaged by a vortex tube entering the stagnation zone is sharply delineated in these two photographs. Furthermore, the outline of the vortex tube in the normal plane reveals plainly that its axis is in the $x_{1}$-direction, i.e., a cross-vortex tube. Subsistence of stretching and a concomitant increase in the angular velocity are suggested by the contours of the smoke filaments. The wrapping of a previous tube around the cylinder further indicates the tilting. Vivid impressions of these flow features are particularly imparted by the blown-up image.

A coherent substructure of standing vortices near the stagnation zone, as pictured in Fig. 4, is advanced by the vorticity-amplification theory. One consequently expects that the visualized flow pattern in the normal plane will generally exhibit a similar vortex structure at all times. Top-view motion pictures were taken in order to assert this persisting occurrence of the contemplated cellular flow pattern. A sequence of 20 stills from 
a selected movie strip $833-1 / 3 \mathrm{~ms}$ long and their edited sketches are displayed in Figs. 11.1 to 11.5. In these figures $T$ stands for the top view, and the scale of the schematics is $6: 5(6 \mathrm{~mm}=$ $5 \mathrm{~mm}$ ). Examination of this sequence was generally conducted in a similar manner as for the side views. The permanency of the coherent vortex structure in the normal plane precluded the interpretation of the flow events in terms of distinct growth phases as in the case of the side-view sequence. In fact, these phases can only be unraveled when the cross-vortex tube is observed in the stream plane (side views in the $\mathrm{x}_{1} \mathrm{x}_{2}$-plane). This is due to the three-dimensionality of the cross-vortex tubes. As a result the analysis of the top-view sequence focused on ascertaining the significant scales of the standing cross-vortex tube and the

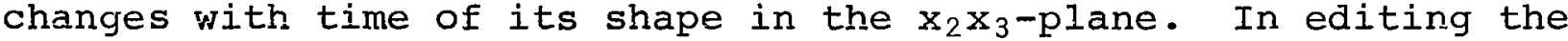
sketches emphasis was placed on tracking representative pathlines (or contour lines) within the cross-vortex tube. Additionally, the smoke streamers swept over and underneath the cylinder were traced. In the schematics the former is marked by a double broken line (--.-) while the latter is delineated by a simple broken Iine (-*-). These smoke filaments represent the tilting and subsequent winding of cross-vortex tubes around the cylinder.

an outstanding illustration of the tubular flow pattern near the stagnation zone is rendered by the top-view sequence shown in Figs. 11.1 to 11.5. Particularly revealing is the striking persistence of the standing cross vortex whose axis is clearly in the $\mathrm{x}_{1}$-direction and with its core distinctively outside the laminar boundary layer. These features are exhibited by the vortex flow pattern cver its entire time span of 833-1/3 ms. In approximating this life cycle the guiding criterion was the tracking of the successive changes in the pathlines starting with a reasonably defined regular vortex pattern close to the body until the recurrence of a roughly similar pattern at about the same spatial

position. In comparing the images and their schematics displayed in frames $1-\mathrm{T}$ at $t=41-2 / 3 \mathrm{~ms}$ (Fig. 1l.I) and 20-T at $t=833-$ $1 / 3 \mathrm{~ms}$ (5ig. 11.5) the proposed estimate for the life cycle is substantiated. In these two particular frames which are 791-2/3 ms apart, the contour lines of the engulfed smoke are similar in their almost regular pattern and the leading edge of both vortex tubes is at roughly the same distance of $5 \mathrm{~mm}(0.20 \mathrm{in})$ upstream of the cylinder. Time spans of nearly the same length within \pm 5 to 108 were deduced from all the other top-view sequences using the same guiding criterion. A life cycle of about $800 \mathrm{~ms}$ is consequently advanced with regard to the evolution of the vortex structure in the normal plane. It is interesting to point out that the side-view sequence indicated a life cycle of roughly 700 to $750 \mathrm{~ms}$ as regards the growth of a cross-vortex tube. One can hence assume that the life cycle of a stretched cross-vortex tube from its onset near the stagnation zone until its full blending into the boundary layer extends over a time period of about 700 to $800 \mathrm{~ms}$. This time span applies to the particular Reynolds number 
$\left(8 \times 10^{3}\right)$ of this flow situation. Shorter life cycles are expected with increasing Reynolds numbers.

The most prominent feature disclosed by the top views is that of the almost quasi-ordered cellular flow pattern. Within the entire life cycle of $833-1 / 3 \mathrm{~ms}$ the consistency of the vortex flow structure is most remarkable. In examining both the images and their sketches a rapid decrease in the scale of the cross-vortex tube across and down its "barrel"-i.e., along its axis in $x_{1}$ direction-is readily perceived. This diminution in the scale must be accompanied by an augmentation in the angular velocity according to conservation of angular momentum. A gradual increase in the smoke rotational velocity is, in fact, vividly perceivable elsewhere in the motion picture. The continuous scale decrease and the accompanying increase in the rotational velocity along the shrinking pathlines that are observed at all times attests to the preferred stretching experienced by the cross-vortex tubes.

Scales of selected pathlines were further derived from the interpretative sketches according to the prescribed 6:5 scale. The estimates of these streamwise spatial scales-i.e., the scales in the $\mathrm{x}_{2}$-direction-are marked on all the schematics in Figs. 11.1 to 11.5. They ranged from about 2 to $32 \mathrm{~mm}(0.08$ to $1.26 \mathrm{in})$. These scales are solely coarse approximations but, nevertheless, they are indicative of the size of the standing vortices near the stagnation zone. It is further significant to remark that these scales are of the same order of magnitude as their counterparts in the $\mathrm{x}_{1} \mathrm{x}_{2}$-plane (the stream plane) which represent the width of the vortex tubes. These consistent scales of the vortex tubes substantiate the predicted coherent substructure of a regular array of cross vortices. Generally, the scales are greater than the neutral wavelength which is $5.6 \mathrm{~mm}$ (see sect. 2). The scales smaller than the neutral one are likely to outline cross-vortex tubes in their poststretching phase. A similar situation was identified in the stream plane. Assessment of the most amplified scale directly from the sketches is not feasible since its determination is contingent upon knowing the turbulent energy distribution. The important aspect of the observed scale structure is that cross vorticity wl undergoing stretching and, therefore, amplification is prevalent near the stagnation zone. As a matter of fact, the vortex structure visualized in the normal plane complements and corroborates the flow picture rendered by the side views in the stream plane.

Visualization of the interaction between the amplified turbulence and the laminar boundary layer is not fully attainable in the top views owing to their view angle. However, one can at least observe in the normal plane the penetration of the largescale vorticity into the stagnation laminar boundary layer. It is discerned starting from $t=125 \mathrm{~ms}$ (frame $3-\mathrm{T}$ in Fig. 1l.1) and it is seen to last until $t=625 \mathrm{~ms}$ (frame 15-T in Fig. 11.4) in the life cycle. This time span of about $500 \mathrm{~ms}$ is generally 
consistent with its counterpart deduced from the side views. The penetrating large scale is essentially the leading edge of the standing cross-vortex tube. It is further important to note that the tilting and wrapping of vortex tubes around the cylinder is represented in the top views by the smoke streamers carried over and beneath it. These smoke streamers are consistently detected in al1 the frames shown in Figs. 11.1 to 11.5. The interaction of these winding vortex tubes with the laminar boundary layer and their crucial role in generating and sustaining a turbulent boundary layer were unveiled by the side views.

An additional feature of utmost significance hitherto unexplained is unfolded by the top images. In comparing the shape of the cross-vortex tube at times $t=41-2 / 3 \mathrm{~ms}$ (frame $1-\mathrm{T}$ in Fig. 1l.1) and $t=208-1 / 3 \mathrm{~ms}$ (frame 5-T in Fig. 11.2) in its life cycle, it is evident that its initial regular form experiences distinct distortion. With increasing time the contortions suffered by the cross-vortex tube become more severe. The successive stages in the development of this warping are perceived until $t=$ 791-2/3 $\mathrm{ms}$ (frame 19-T in Fig. 11.5), and, hence, the deformation of the cross-vortex tube persists during most of its life cycle (833-1/3 ms). In scrutinizing frames 5-T to 19-T and their respective sketches (Figs. 11.2 to 11.5 ) one can clearly detect that the distortion is primarily sustained by the large scale of the crossvortex tube while its smaller scales are much less affected. This distortion of the large scale is revealed by its pronounced embaying and denting. According to the vorticity-amplification theory standing cross-vortex tubes of the same scales are regularly distributed along the span of the stagnation zone as portrayed in Fig. 4. Within the cells of this spanwise array the rotation repeatedly changes its direction. Consequently, a continuous inter-cell interaction is expected which can undoubtedly cause deformations in the shape of each standing cross-vortex tube. It is therefore conjectured that the observed strong contortions of the cross-vortex tube are induced by its interaction with its two adjacent tubes. Based on the continuously changing pattern of the embayments and dents of the interface, viz., the large scale, it is apparent that the rotation within both contiguous left and right tubes is in a counterclockwise direction. These two tubes are in turn distorted by the one located in between them within which the rotation is in a clockwise direction as perceived in the displayed stills and sketches. For the sake of illustrating the proposed inter-vortex interaction mechanism the envisioned two adjacent standing cross-vortex tubes are depicted by dashed lines in sketch 10-T (Fig. 11.3). At this time no theoretical model for this interaction has yet been put forth. It is therefore significant to stress the invaluable role played by the visualization in detecting the inter-vortex interaction and, moreover, in supplying the physical insight for advancing a sensible hypothesis concerning its occurrence. 
To further demonstrate the subsistence of a coherent vortex substructure along the stagnation zone and the subsequent winding of the stretched cross-vortex tubes over the cylinder, motion pictures were shot when smoke was injected in exactly the same way at two stations $15.24 \mathrm{~cm}$ (6 in) apart. These top views were taken directly above the cylinder in order to include the flow pattern above it within the depth of field. A representative sequence of 8 frames extending over a time interval $t=333-1 / 3 \mathrm{~ms}$ along with their corresponding edited sketches is given in Figs. 12.1 and 12.2. In these figures $T / D$ designates the top view of the double-smoke injection, and the scale of the schematics is $1: 4$ ( 1 $\mathrm{mm}=4 \mathrm{~mm}$ ). The entrainment of both smoke filaments by similar cross-vortex tubes near the stagnation zone is startlingly perceived in all the stills. In fact, the images of both vortex flow patterns are nearly identical. Thus, a coherent array consisting of regularly distributed cross-vortex tubes of about the same scales, as portrayed in Fig. 4, exists along the span of the stagnation zone.

Worthy insights into the wrapping of stretched cross-vortex tubes over the cylinder were further supplied by this top-view sequence of the double-smoke injection. In sketching the flow pattern the delineation of the large scale (or envelope) of the cross-vortex tubes drawn over the cylinder was stressed. This envelope is outlined on the schematics by broken lines (-・-). On the left side of all the stills the winding over the cylinder of a developed cross-vortex tube is noticed. This winding essentially represents the streamwise tilting of a stretched crossvortex tube viewed in the $\mathrm{x}_{2} \mathrm{x}_{3}$-plane. Particularly notable is the persistent shape of the tube envelope during the entire time span of $333-1 / 3 \mathrm{~ms}$ which is distinctly observed in the sketches displayed in Figs. 12.1 and 12.2. Inspection of the smoke pattern observed on the right side of the stills unravels the development of the tube winding. The outset of the pulling of a cross-vortex tube is discerned on the right side of the first still in this sequence, viz., frame 1-T/D in Fig. 12.1. In tracking this tube one notices the continuous growth of its wrapping with increasing time which becomes fully perceivable after about $166-2 / 3$ ms as seen in frame $5-T / D$ and in the subsequent frames given in $F i g$. 12.2. Once this drawn tube is developed, its general shape is similar to that of the tube on the left side of the stills. Both tubes exhibit kinks and slight bendings which are likely to be induced by their interaction with adjacent vortex tubes. The envelopes of both tubes were next estimated from the sketches according to the 1:4 scale used. Their widths in the normal plane ranged from about 16 to $40 \mathrm{~mm}(0.63$ to $1.6 \mathrm{in})$. These scales are consistent with their counterparts in the $x_{1} x_{2}$-plane derived from the side views. The amplified cross-vorticity is contained within these winding cross-vortex tubes. This is the agent responsible for the breeding and the maintenance of the turbulent boundary layer which was distinctively revealed by the side views. The significant aspects regarding the winding of stretched cross-vortex 
tubes over the body are their spanwise coherent structure and their continuous subsistence.

\subsection{Rear Views}

To fully consumate the visualization of the flow events in all the three planes, several motion pictures were shot from a rear view for obtaining a picture of the flow pattern projected in the $x_{1} x_{3}$-plane or the cross plane (see Fig. 3). The viewpoint was looking upstream into the oncoming flow from a position downstream and slightly above the cylinder. In making these motion pictures the goal was to observe the tilting around the cylinder of the smoke engulfed by a stretched cross-vortex tube, the interaction of the amplified vorticity (or turbulence) with the laminar boundary layer and, finally, the ensuing turbulent boundary layer. A representative sequence of 16 stills from a movie strip extending over a time span of $666-2 / 3 \mathrm{~ms}$ and their interpretative schematics are shown in Figs. 13.1 to 13.4. In these figures $R$ stands for rear view, and the scale of the sketches is $3: 10(2 \mathrm{~mm}=10 \mathrm{~mm})$. The laminar boundary layer delineated in the schematics corresponds to an angle of about 80 to $90^{\circ}$ (measured from the stagnation point) owing to the rear viewpoint. It is consequently slightly thicker than at the stagnation zone considering its downstream growth along the cylinder circumference. A thickness of about 6 $\mathrm{mm}(0.24 \mathrm{in})$, as in the side-view schematics, is therefore shown in all the sketches.

Inspection of this sequence was carried out in the same way as for the side and top views. The time span or life cycle of the rear-view sequence, viz., $666-2 / 3 \mathrm{~ms}$, was selected in order to track the evolution of a cross-vortex tube from the beginning of its winding over the cylinder until the full manifestation of a distinct spiral pattern which indicates, in turn, the growth of a turbulent boundary layer. In tracing out the sketches of the smoke flow patterns projected in the cross plane the accent was consequently put on outlining the pathlines of the tilting crossvortex tube and the subsequent spiraling of the smoke filaments over the cylinder. The turbulence-generating grid and the smoke injection tube are recognized in the background of the stills to the right of the smoke filaments. These two details are not included in the schematics.

Scrutiny of the rear-view sequence unravels, in the background of the stills $1-R$ to $9-R$ shown in Figs. 13.1 to 13.3 , a persistently coherent vortex flow pattern. This pattern is the image in the cross plane of an oncoming cross-vortex tube that undergoes gradually increasing tilting. In tracing the sketches of the smoke flow pattern within the time span of these nine frames ( 375 msl, the distorted smoke filaments over the cylinder were disregarded. The reason for this was the set aim to track the tilting 
of an incoming cross-vortex tube and its subsequent dispersion into a spiral flow. In assessing the tilting the yardstick was the perceived direction of the axis of the visualized cross-vortex tube. Tilting around a cylinder has to lead to the shifting of the axis of a cross-vortex tube from its initial cross direction ( $x_{1}-$ direction) to the streamwise direction ( $x_{2}$-direction). In other words, the axis of a cross-vortex tube no longer remains parallel to the $x_{1}$-direction as the tilting acts. It is therefore expected to observe in the $x_{1} x_{3}$-plane a vortex tube with its axis progressively pointing in the streamwise $x_{2}$-direction. This tilting effect is revealed by the smoke vortex pattern perceived in the background of the images $2-\mathrm{R}$ to $9-\mathrm{R}$ and sharply outlined elsewhere in the motion pictures. In comparing the shape of the pathlines in sketches $1-R$ and $2-R$ ( $t=41-2 / 3$ and $83-1 / 3 \mathrm{~ms}$ in Fig. 13.1) one can discriminate the beginning of the tilting. In sketch $1-R$ the axis of the cross-vortex tube is apparently in the $\mathrm{x}_{1}$-direction. On the other hand, the pattern of the contour lines exhibited in sketch 2-R indicates the inception of the tilting since the axis of the cross-vortex tube is turning in the $\mathrm{x}_{2}$-direction.

Examination of the persistent vortex pattern of pathlines in the cross plane depicted in the subsequent sketches 3-R to 9-R $(t=125$ to $375 \mathrm{~ms}$ in Figs. 13.1 to 13.3 ) unfolds the progress of the tilting. The tubular shape of the contour Iines becomes more pronounced in the $x_{1} x_{3}$-plane with increasing time. This gradual realization of a sharp vortex pattern in the cross plane underscores the continuous veering of the cross-vortex tube axis away from its original cross direction ( $x_{1}$-direction) to the downstream direction ( $x_{2}-$ direction). Starting from $t=208-1 / 3 \mathrm{~ms}$ in the life cycle (frame 5-R in Fig. 13.2) the pointing of the vortextube axis directly in the streamwise direction is inescapably perceived. This is the measure indicating the attainment of the final tilted position of the stretched cross-vortex tube. Thus, the streamwise tilting of stretched cross-vortex tubes, as pictured in Figs. 2 and 3 and as observed in the stream plane $\left(x_{1} x_{2}-\right.$ plane), is further demonstrated by the flow pattern in the $\mathrm{x}_{1} \mathrm{x}_{3}-$ plane.

Approximation of the scales of the tilted vortex tube-i.e., the lateral scales in the $x_{3}$-direction-was next carried out according to the $3: 10$ scale of the schematics. These lateral spatial scales of the pathlines ranged from about 7 to $42 \mathrm{~mm}(0.28$ to $1.65 \mathrm{in}$ ) as shown in the sketches $2-\mathrm{R}$ to $9-\mathrm{R}$. They are generally of the same magnitude as their counterparts estimated in both the stream and normal planes $\left(x_{1} x_{2}-\right.$ and $\left.x_{2} x_{3}-p l a n e s\right)$. In the former plane the width of the cross-vortex tube varied from 4 to $40 \mathrm{~mm}$ (see Figs. 9.1 to 9.5) while its streamwise scale and envelope in the latter plane (see Figs. 11 and 12) ranged from 2 to $32 \mathrm{~mm}$ and from 16 to $40 \mathrm{~mm}$, respectively. In fact the tube envelope in the normal plane shown in Figs. 12.1 and 12.2 is the counterpart of the large lateral scale in the cross plane. This remarkable 
consistency of the vortex-tube spatial scales in all the view planes clearly indicates the subsistence of a coherent vortex flow structure.

Further inspection of the time evolution of the flow events in the cross plane reveals an expansion of the scales of the tilted cross-vortex tube followed by its interaction with the laminar boundary layer and the subsequent development of a turbulent boundary layer. In examining the contour lines of the tilted vortex tube within the time interval $t=41-2 / 3$ to $333-1 / 3 \mathrm{~ms}$ (frames 2-R to $8-\mathrm{R}$ in Figs. 13.1 and 13.2) one can distinctly apperceive their continuous widening. For instance, the large lateral scale increases from about 24 to $42 \mathrm{~mm}$ ( 0.94 to 1.65 in) within this time period. A similar expansion with increasing time was observed in the stream plane (side view) with regard to the gross scale of the winding cross-vortex tube. Vorticity is exchanged among its components according to the vorticity-velocity gradient interaction (see Eq. (1)) during the tilting. This exchange of vorticity represents essentially an inviscid interdiffusion of vorticity. In the organized streamrise tilting characteristic to the stagnation flow, the amplified vorticity within the vortex tube diffuses outward inducing its own distention. Thus, the observed scale increase of the vortex tube represents the manifestation of its tilting: The outward diffusion of vorticity and the accompanying scale widening lead furthermore to the gradual disintegration of the coherent structure of the vortex tubes. Simultaneously, the diffused vorticity (or turbulence) fosters the development of a turbulent boundary layer which, in turn, promotes the breakup of the incoming coherent vortex tubes.

The flow evolution disclosed by frames $8-\mathrm{R}$ to $16-\mathrm{R}$ (t $=$ $333-1 / 3$ to $666-2 / 3 \mathrm{~ms}$ ) shown in Figs. 13.2 to 13.4 by and large lends support to the conjectured mechanism regarding the generation of a turbulent boundary layer. Penetration of the tracked vortex tube into the laminar boundary layer is first detected at about $t=333-1 / 3$ to $375 \mathrm{~ms}$ (frames $8-R$ and $9-R$ ) and it is seen to continue throughout the entire life cycle until $t=666-2 / 3$ $\mathrm{ms}$ (frame 16-R). The beginning of the interaction is unveiled by the invasion of the large-scale vorticity into the laminar boundary layer which is ciscerned in frames 8-R and 9-R. A similar picture of the interaction was rendered by the top views in the $\mathrm{x}_{2} \mathrm{x}_{3}-$ plane (the normal plane). Starting from frame 10-R $(t=416-2 / 3$ ms in Fig. 13.3) the engulfment of the smoke filaments by a continuously growing spiral pattern is clearly recognized. Most preeminent are the lateral spreading and the streamwise distention of the spiraling with increasing time exhibited by the pathlines depicted in sketches $10-R$ to $16-R(t=416-2 / 3$ to $666-2 / 3 \mathrm{~ms})$. A similarly distorted flow pattern over the cylinder is observed in frames $1-R$ to $8-R$ (Figs. 13.1 and 13.2). Recall that this flow pattern was not traced considering the aim of the examination of the rear-view sequence. 
The persistent spiral flow pattern over the cylinder indicates the development of a turbulent boundary layer. Its generation and sustenance is promoted by the continuous diffusion of incoming amplified cross vorticity within the tilted vortex tubes. It is of utmost significance to notice that a similar time evolution of the flow events was deduced from the side views in the stream plane. The development of a turbulent boundary layer as a result of the tilting over the cylinder of stretched cross-vortex tubes, as manifested in the stream plane, is further indicated by the flow pattern in the cross plane. 


\section{DISCUSSION OF RESULTS}

Comprehensive insights into the flow pattern were rendered by still photographs and, particularly, by motion pictures taken from three differen't viewpoints, viz., side, top and rear view angles. Frame by frame examination of selected movie sequences extending over time intervals of up to $833-1 / 3 \mathrm{~ms}$ (20 frames) led, despite its limitations, to the acquisition of a reasonably quantitative interpretation of the gross flow structure in addition to supplying an in-depth qualitative apperception of the flow pattern. This frame by frame inspection warranted the securing of interpretative schematics of the flow patterns delineated by the entrained smoke filaments. In carrying out the analysis of the movie frames and in deriving their interpretative sketches of the pathlines, emphasis was placed on tracking the development of a cross-vortex tube. As a result, its temporal and spatial evolution was outlined in the stream, normal and cross planes, viz., from side, top and rear viewpoints. This threefold visualization furnished a remarkable overall picture of the salient traits of the flow.

The time history (or life cycle) of a cross-vortex tube since its emergence near the stagnation zone until its dispersion within the growing turbulent boundary layer was traced in the stream plane. Embryonic, maturing and aging phases in the development of the tracked cross-vortex tube were identified. The most outstanding illustration of a standing vortex structure near the stagnation zone and of its evolution was supplied by the top views in the normal plane. Engulfment of smoke filaments by a sharply defined tubular flow pattern was steadfastly discriminated. Subsistence of distinct cross-vortex tubes with their cores outside the laminar boundary layer was further persistently discerned in the side and, particularly, in the top views. Based on the temporal evolution of the tracked cross-vortex tube observed in the stream and normal planes, its life cycle was estimated to extend over a time span of about 700 to $800 \mathrm{~ms}$.

The stretching of the cross-vortex tube was strikingly revealed by the continuous and rapid decrease of its scales across and down its barrel (along its axis) and by the concurrent increase in the smoke rotational velocity. This flow picture was vividly perceivable in the side- and top-view motion pictures and it was detectable in the stills of the selected frames. Representative scales of the tracked cross-vortex tube deduced from its pathlines depicted in the interpretative sketches are further indicative of the occurrence of the stretching. They ranged from about 4 to 40 $\mathrm{mm}$ and from roughly 2 to $32 \mathrm{~mm}$ in the stream and normal planes, respectively, while the neutral scale was only $5.6 \mathrm{~mm}$. Thereby cross vorticity at scales larger than the neutral wavelength undergoing stretching and, consequently, amplification prevails near the stagnation zone. This vorticity intensification leads in turn to the predicted amplification of the streamwise turbulence. 
The revealing consistency of the scale structure of the traced cross-vortex tube attested to the realization of a coherent vortex flow structure near the stagnation zone. Further evidence that this quasi-ordered vortex structure consists of repetitive cells of roughly the same scales within which the rotation alternates in its direction was unfolded by the top-view sequence. Distinct distortions of the large-scale of the outlined crossvortex tube, hitherto unexplained, were consistently observed. Such contortions can only be induced by the interaction of this tube with two adjacent similar cross-vortex tubes in which the rotation is in the opposite direction. An additional demonstration of the existence of a coherent array of spanwise distributed cells was furnished by the simultaneous visualization of two cross-vortex tubes at a certain distance apart. Both tubes exhibited a startlingly similar structure and, moreover, their envelope (or gross scale) was by and large of the same magnitude. They ranged from about 16 to $40 \mathrm{~mm}$ and, hence, they are consistent with their counterparts deduced from the other side- and top-view sequences. It is thus apparent that the stretching acts as the triggering agent which provokes the realization of a coherent array of cross-vortex tubes near the stagnation zone. This array represents essentially an organized substructure within the turbulent field.

The streamwise tilting of the tracked cross-vortex tube and its winding over the cylinder were best perceived in the side and rear views. Particularly revealing in the cross plane (the rear viewpoint) was the gradual shifting of the tube axis from its initial cross direction to the streamwise direction with increasing time. The final tilted position was observed after a time interval of about $208 \mathrm{~ms}$ in its life cycle. Even during the tilting and the winding the coherent shape of the cross-vortex was preserved. Its scales deduced from the interpretative sketches in the cross plane ranged from roughly 7 to $42 \mathrm{~mm}$. They are thus of the same magnitude as their counterparts observed in the stream and the normal planes. This lends further support to the occurrence of a coherent vortex flow structure near the body stagnation zone.

The interaction of the tracked cross-vortex tube with the laminar boundary layer and the growth of a turbulent boundary layer were distinctly apperceived in all three views. Inception of the interaction at the stagnation zone was discerned in the stream and normal planes at about the same time in the life cycle of the cross-vortex tube, viz., starting at about $125 \mathrm{~ms}$. In the cross plane the interaction was first detected at a later time, viz., at about $333 \mathrm{~ms}$, owing to the rear view-point. The realization of the interaction was revealed by the invasion of the largescale vorticity into the laminar boundary layer as clearly perceived in all three view angles. Essentially, the interaction is the consequence of the penetration into the laminar boundary layer of the amplified vorticity (or turbulence) contained within the winding cross-vortex tube. A continuous supply of turbulence 
warrants the sustenance of the interaction which leads to the growth of a turbulent boundary layer. The needed turbulence is furnished by the outward diffusion of the vorticity concentrated within the incoming vortex tubes. In tracking the evolvement of the interaction, severe distortions of the smoke flow pattern within a continuously distending vortex tube were strikingly discriminated in the side views. The prominent trait of this disordered flow was the spiraling of the smoke filaments which was additionally disclosed by the rear views. A continuous widening of the winding cross-vortex tube followed by its gradual distention into a growing spiral pattern was, moreover, observed in the cross plane (rear view). This scale expansion suggests an outward diffusion of vorticity which is the agent responsible for the fostering of a turbulent boundary layer. Thus, the observed flow pattern clearly indicates the growth of a turbulent boundary layer. 


\section{CONCLUSIONS}

An extensive visualization study of the flow around a circular cylinder was conducted. This study was carried out for the sake of obtaining an overall physical confirmation of the explanation advanced by the vorticity-amplification theory with regard to the amplification of turbulence in stagnation flow and its effects upon the body boundary layer. The visualization was performed at a Reynolds number of $8 \times 10^{3}$ using white smoke composed of titanium dioxide. Turbulence was superimposed in a controlled manner at scales larger than the neutral wavelength of the vorticityamplification theory.

The visualization provided significant evidence verifying: (1) the selective stretching of cross-vortex tubes which is responsible for the amplification of cross vorticity and, hence, of streamwise turbulence; (2) the streamwise tilting of stretched cross-vortex tubes; (3) the existence of a coherent vortex flow structure near the stagnation zone; (4) the interaction of the amplified vorticity with the laminar boundary layer; and, finally, (5) the development of a turbulent boundary layer. Hence, the visual observations are lending substantial support to the flow development put forward by the vorticity-amplification theory. 


\section{APPENDIX A}

\section{LIST OF SYMBOLS}

D

d

$\mathrm{n}$

R

$\operatorname{Re}_{D}$

S

$t$

$\mathrm{U}_{\mathrm{i}}$

$\mathrm{U}_{2}$

$u_{2}$

$x_{i}$

$\delta$

$\varepsilon_{i j k}$

$\lambda$

$\lambda_{\mathrm{m}}$

$\lambda_{0}$

$\lambda_{\mathrm{S}}$

v

$w_{i}$

Superscripts

o

I

I

1 cylinder diameter

rod diameter

frequency

cylinder radius

Reynolds number for the cylinder

Strouhal number

time

total velocity

streamwise total velocity

streamwise turbulent velocity

Cartesian coordinates

boundary-layer thickness

third-order unit alternating tensor

scale

most amplified scale

neutral scale

Strouhal scale

kinematic viscosity

vorticity

means "initial station"

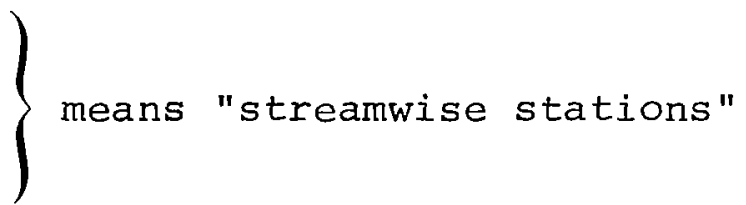


denotes vector

Subscripts

$i, j, k$

$\infty$

\section{Abbreviations}

CSU

FPS

I

$\mathrm{N}$

R

S

T

$T / D$
Cartesian tensor coordinates

freestream

Colorado State University

frames per second

laminar boundary layer

frame counter

rear view in sect. 4.3

side view

top view

top view of double smoke injection

The International system (SI) of measurement was used throughout this work. Conversion from SI units to U.S. customary units was carried out to approximately two significant digits. 


\section{REFERENCES}

1. Piercy, N. A. \& Richardson E. G.: The variation of velocity amplitude close to the surface of a cylinder moving through a viscous fluid. Phil. Mag., vol. VI, 1928, pp. 970-977.

2. Piercy, N. A. V. \& Richardson, E. G.: The turbulence in front of a body moving through a viscous fluid. Phil. Mag., vol. IX, 1930, pp. 1038-1040.

3. Kestin, J. \& Maeder, P. F.: Influence of turbulence on the transfer of heat from cylinders. INACA TN $4018,1957$.

4. Sutera, S. P., Maeder, P. F. \& Kestin, J.: On the sensitivity of heat transfer in the stagnation-point boundary layer to freestream vorticity. J. Fluid Mech., vol. 16, part 4, 1963, pp. 497-520.

5. Sutera, S. P.: Vorticity amplification in stagnation-point flow and its effects on heat transfer. J. Fluid Mech., vol. 21, part 3,1965 , pp. 513-534.

6. Sadeh, W. Z., Sutera S. P. \& Maeder, P. F.: Analysis of vorticity amplification in the flow approaching a twodimensional stagnation point. Z. angew. Math. Phys. (ZAMPJ. Appl. Math. \& Physics), vol. 21, fasc. 5, 1970, pp. 699716.

7. Sadeh, W. Z., Sutera, S. P. \& Maeder, P. F.: An investigation of vorticity amplification in stagnation flow. Z. angew. Math. Phys. (ZAMP-J. App. Math. \& Physics), vol. 21, fasc. 5, 1970, pp. 717-742.

8. Kestin, J.: The effect of freestream turbulence in heat transfer rates. Advances in Heat Transfer, vol. 3, Academic Press, Inc., New York, 1966, pp. 1-32.

9. Kestin, J. \& Wood, R. T.: On the stability of two-dimensional stagnation flow. J. Fluid Mech., vol. 44, part 3, 1970, pp. $461-479$.

10. Bearman, P. W.: Some measurements of the distortion of turbulence approaching a two-dimensional bluff body. J. Fluid Mech., vol. 53, part 3, 1972, pp. 451-467.

11. Hunt, J. C. R.: A theory of turbulent flow round twodimensional bluff bodies. J. Fluid Mech., vol. 61, part 4, 1973, pp. 625-706.

12. Traci, R. M. \& Wilcox, D. C.: Freestream turbulence effects on stagnation point heat transfer. AIAA Journal, vol. 13, no. 7, 1975, pp. 890-896. 
13. Reynolds, 0.: An experimental investigation of the circumstances which determine whether the motion of water shall be direct, or sinuous, and of the law of resistance in parallel channels. Phil. Trans : Roy. Soc., vol. 174, 1883, pp. 935-982.

14. Offen, G. R. \& Kline, S. J.: Combined dry-streak and hydrogen-bubble visual observations of a turbulent boundary layer. J. Fluid Mech., vol. 62, part 2, 1974, pp. 223-239.

15. Laufer, J.: New trends in experimental turbulence research. Annual Review of Fluid Mechanics, eds., van Dyke, M. et al., vol. 7, Annual Reviews Inc., Palo Alto, California, 1975, pp. $307-326$.

16. Roshko, A.: Structure of turbulent shear flow: a new look. AIAA Journal, vol. 14, No. 10, 1976, pp. 1349-1357.

17. Hinze, J. O.: Turbulence. 2nd ed., McGraw-Hill Book Co., New York, New York, 1975, pp. 269-271.

18. Roshko, A.: On the development of turbulent wakes from vortex streets. NACA TR 1191, 1954.

19. Taylor, G. I.: The spectrum of turbulence. Proc. Roy. Soc., A, Vol. CLXIV, 1938, pp. 476-490.

20. Hurd, C. W., Chesky, K. P. and Shapiro, A. H.: Influence of viscous effects on impact tubes. Trans. ASME, J. App. Mech., vol. 20, no. 2, 1953, pp. 253-256.

21. Dean, R. C. Jr., ed.: Aerodynamic Measurements. Gas Turbine Lab., MIT, Cambridge, Massachusetts, 1955, pp. 61-65.

22. Ower, E. \& Pankhurst, R. C.: The measurement of airflow. 4 th ed., Pergamon Press, London, 1966, p. 46.

23. Anon.: Kodak professional black-and-white films. Kodak Publication No. F-5, Eastman Kodak Co., Rochester, New York, 1973.

24. Anon.: Selection and use of Kodak and Eastman motion picture films. Kodak Publication No. H-1, Eastman Kodak Co., Rochester, New York, 1974, pp. 3-5.

25. Schlichting, H.: Boundary-layer theory. 6 th ed., McGrawHill Book Co., New York, New York, 1968, pp. 154-162. 


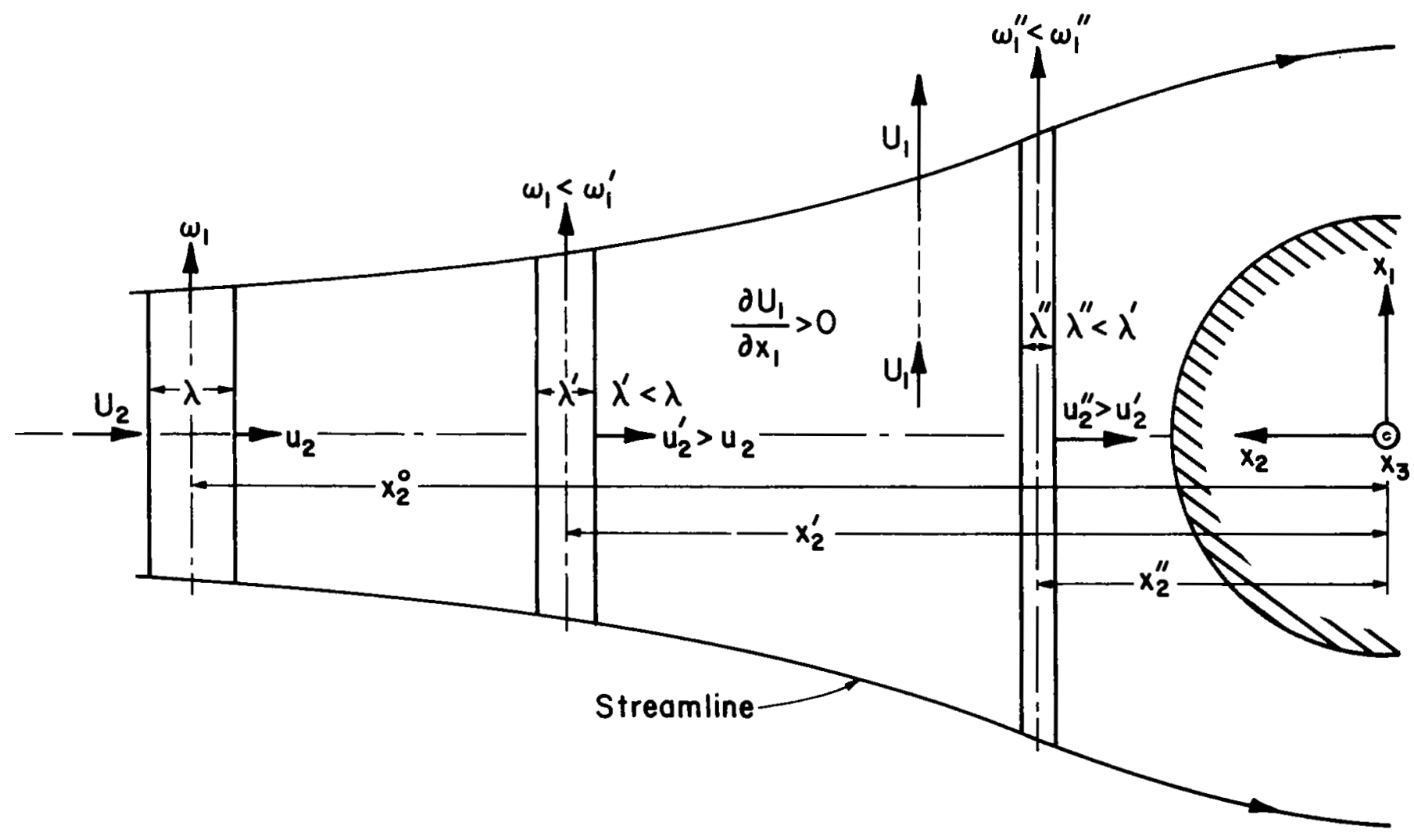

Fig. 1. Stretching of a cross-vortex tube. 


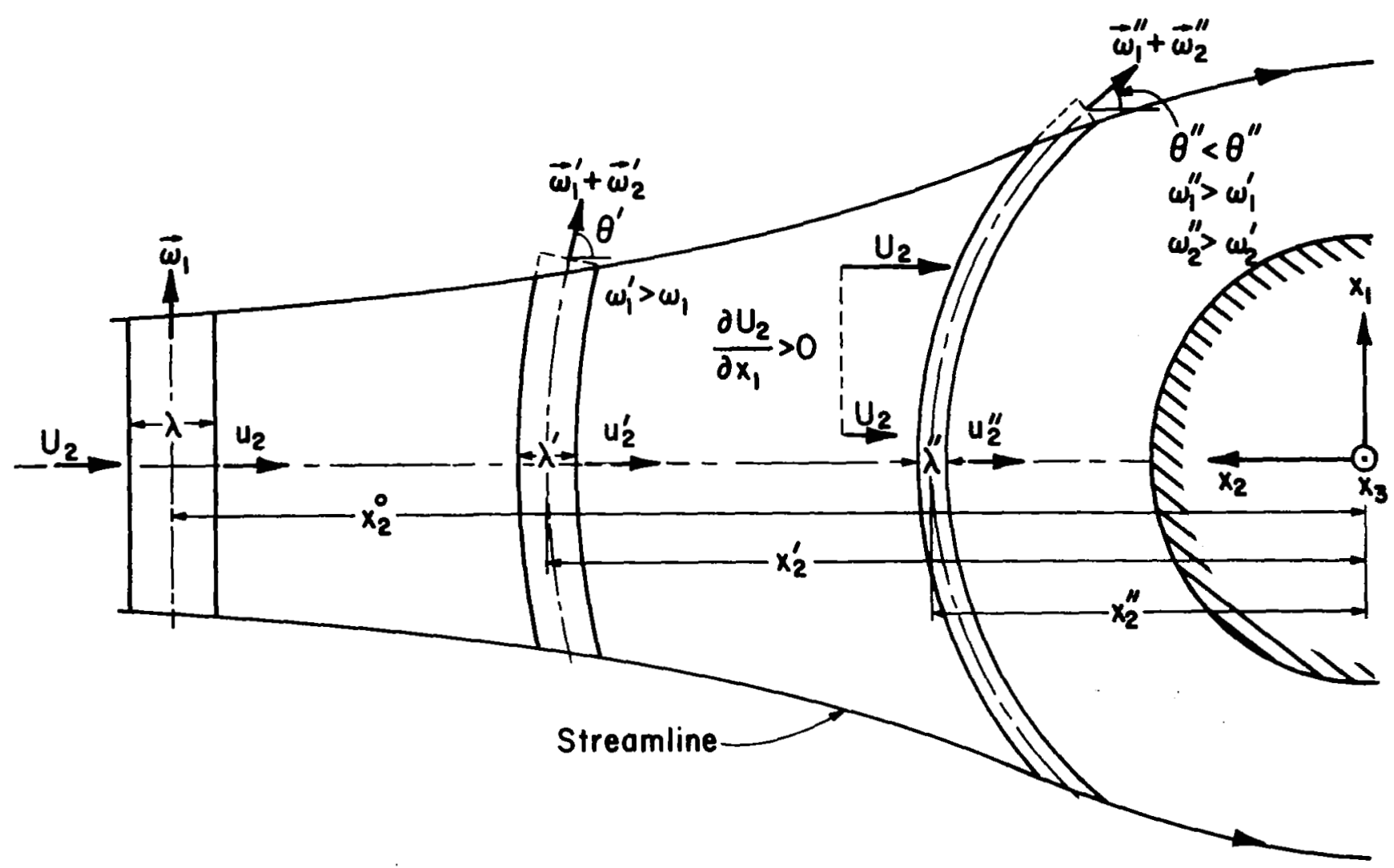

Fig. 2. Tilting of a cross-vortex tube. 


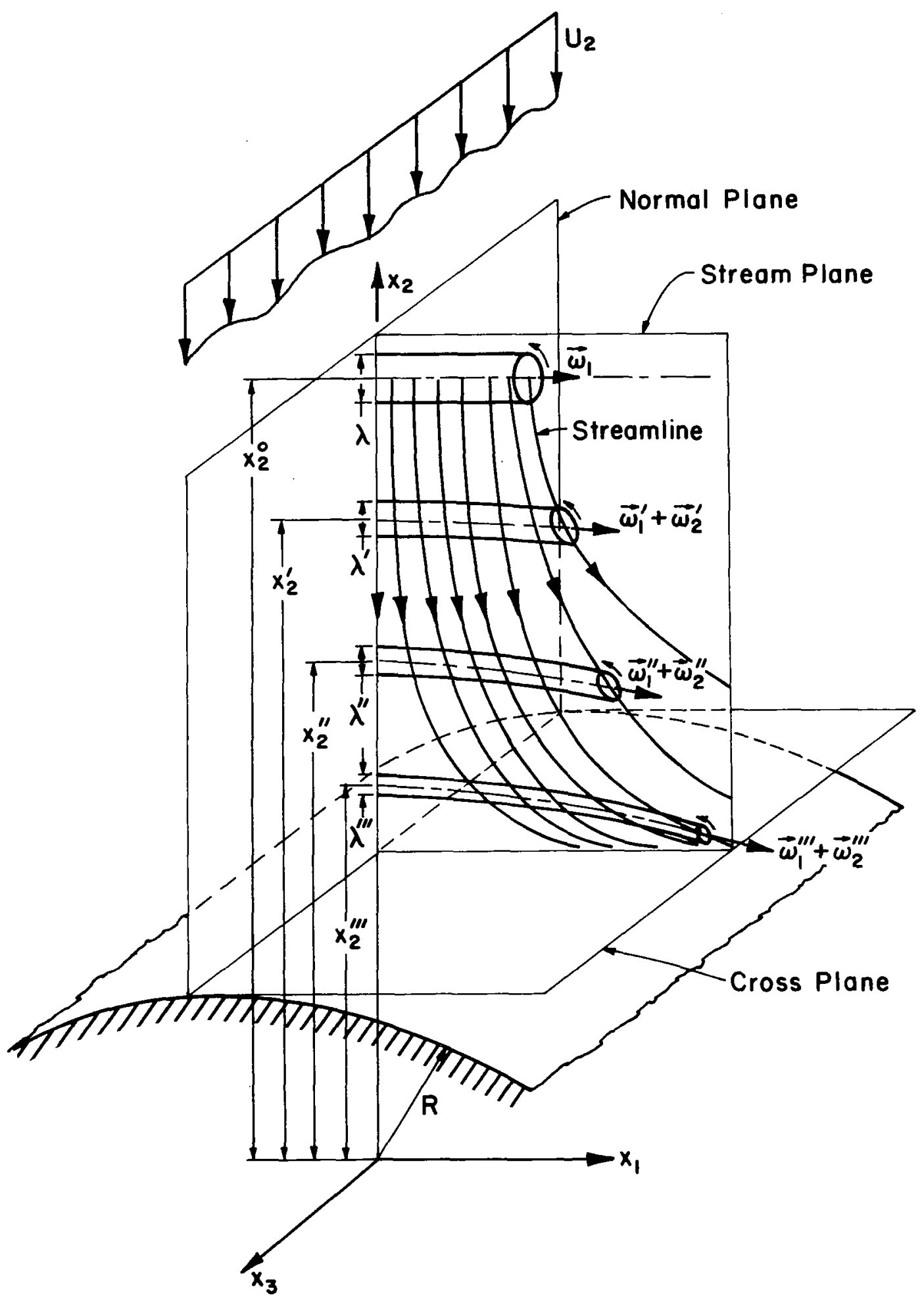

Fig. 3. Illustration of the stretching and tilting of a cross-vortex tube. 


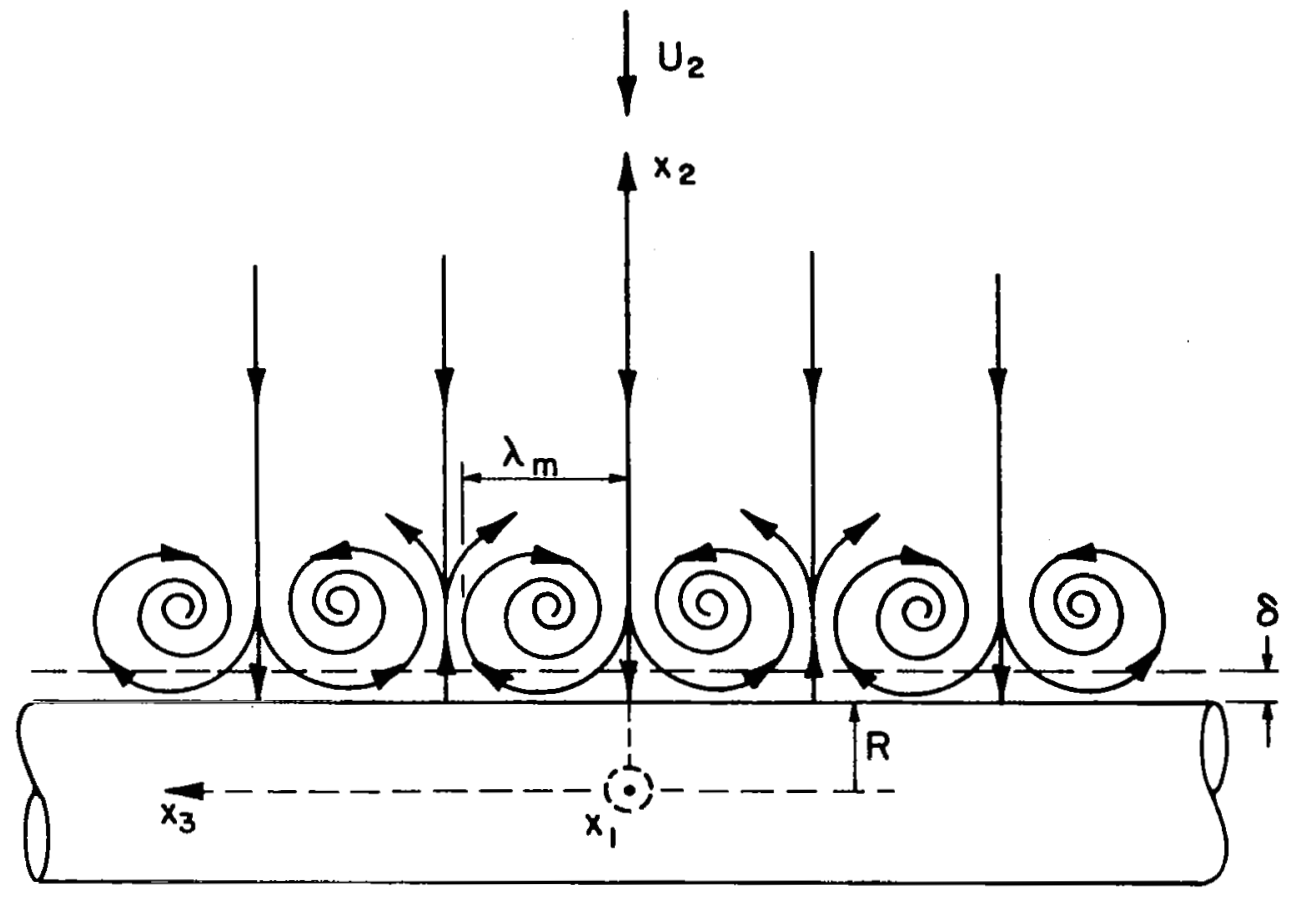

Fig. 4. Theoretical coherent vortex flow pattern near the stagnation zone. 


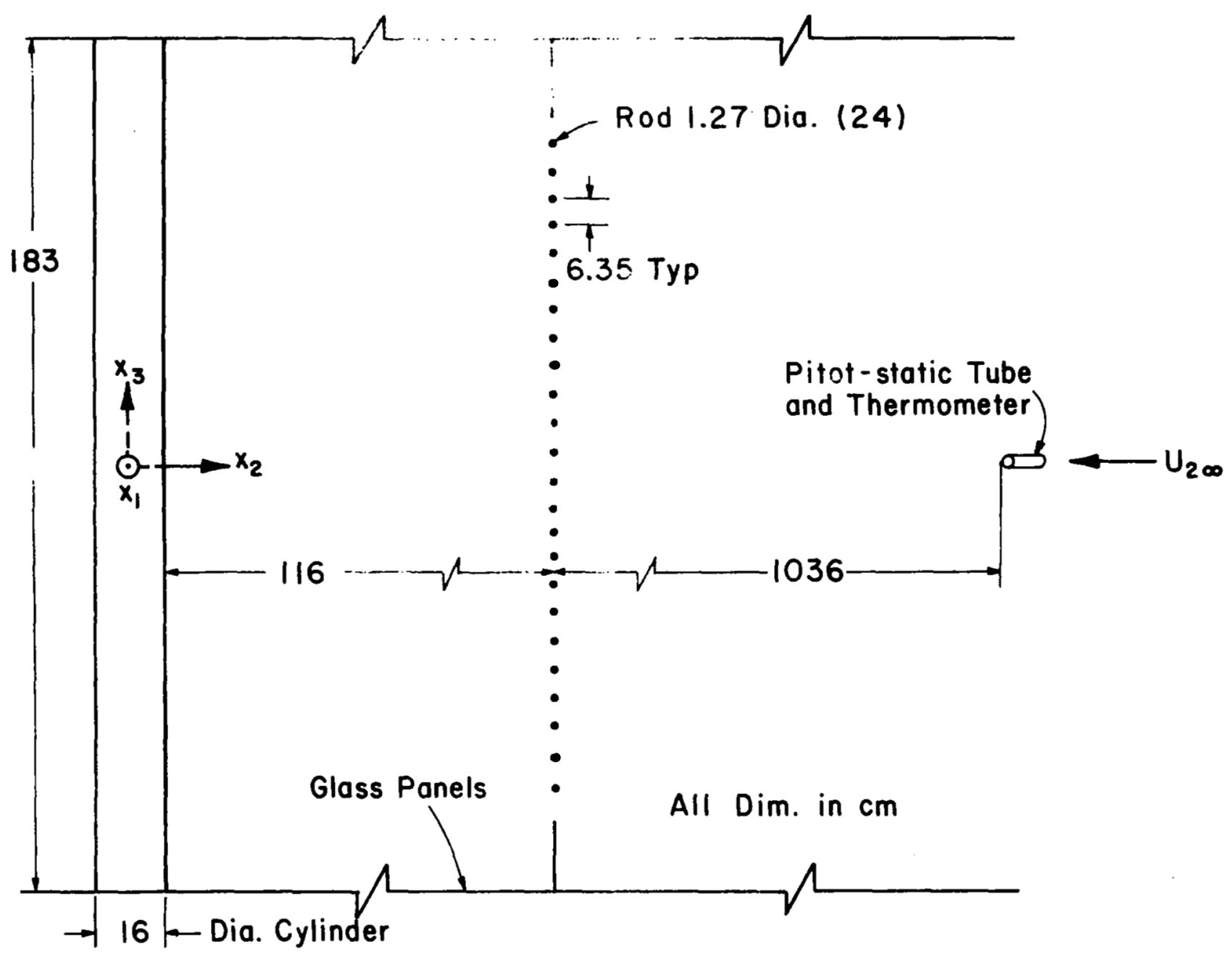

Fig. 5. Sketch of experimental arrangement in the wind tunnel. 


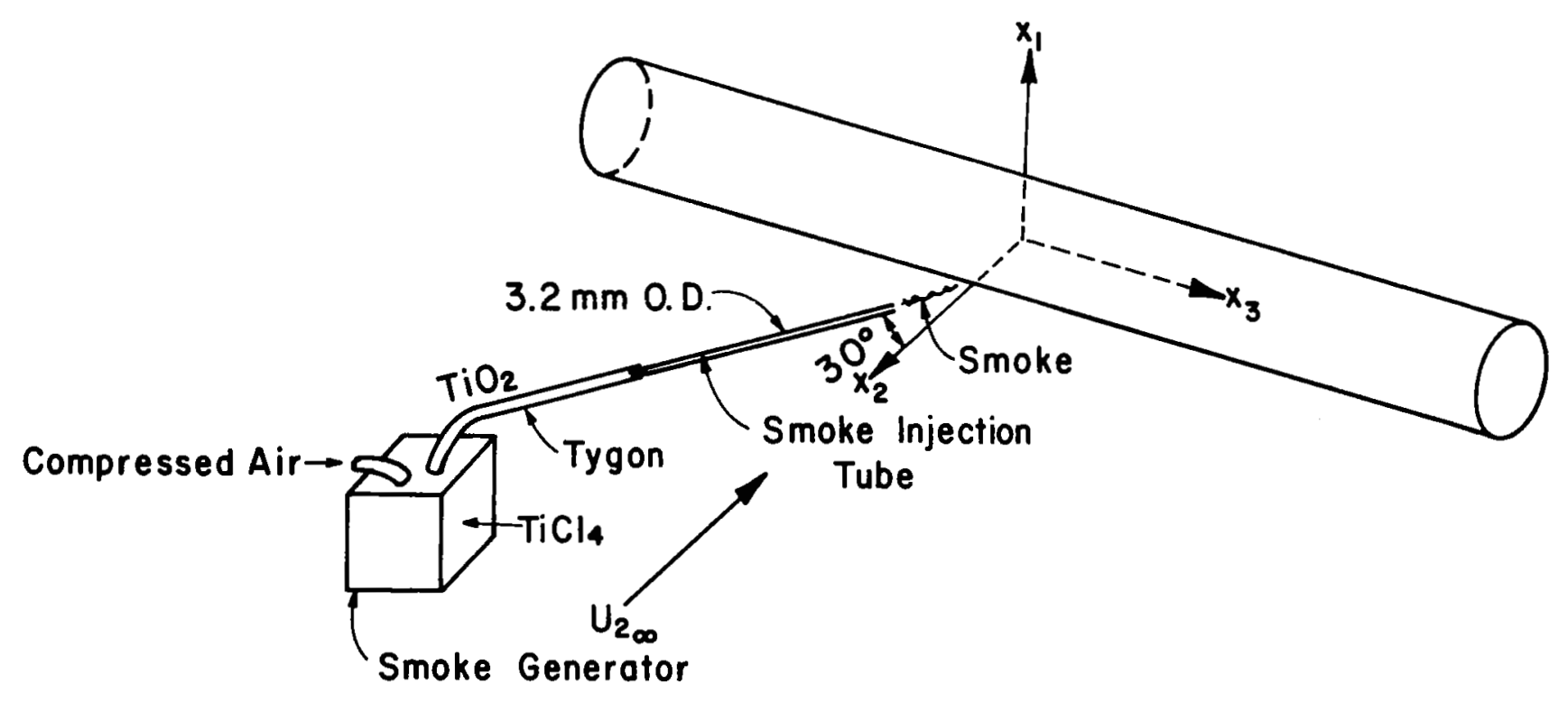

Fig. 6. Smoke injection arrangement. 


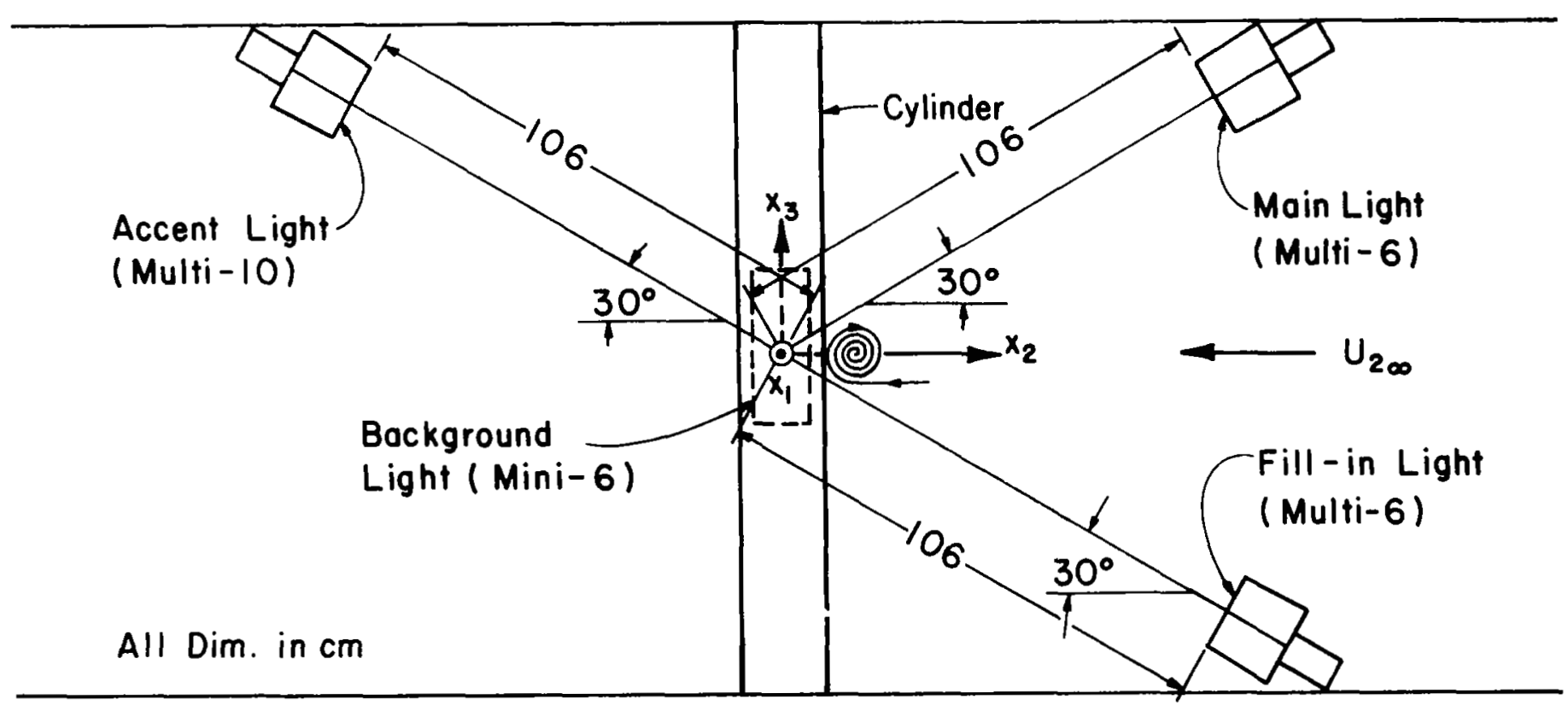

Fig. 7. Typical lighting composition for top-view photography. 

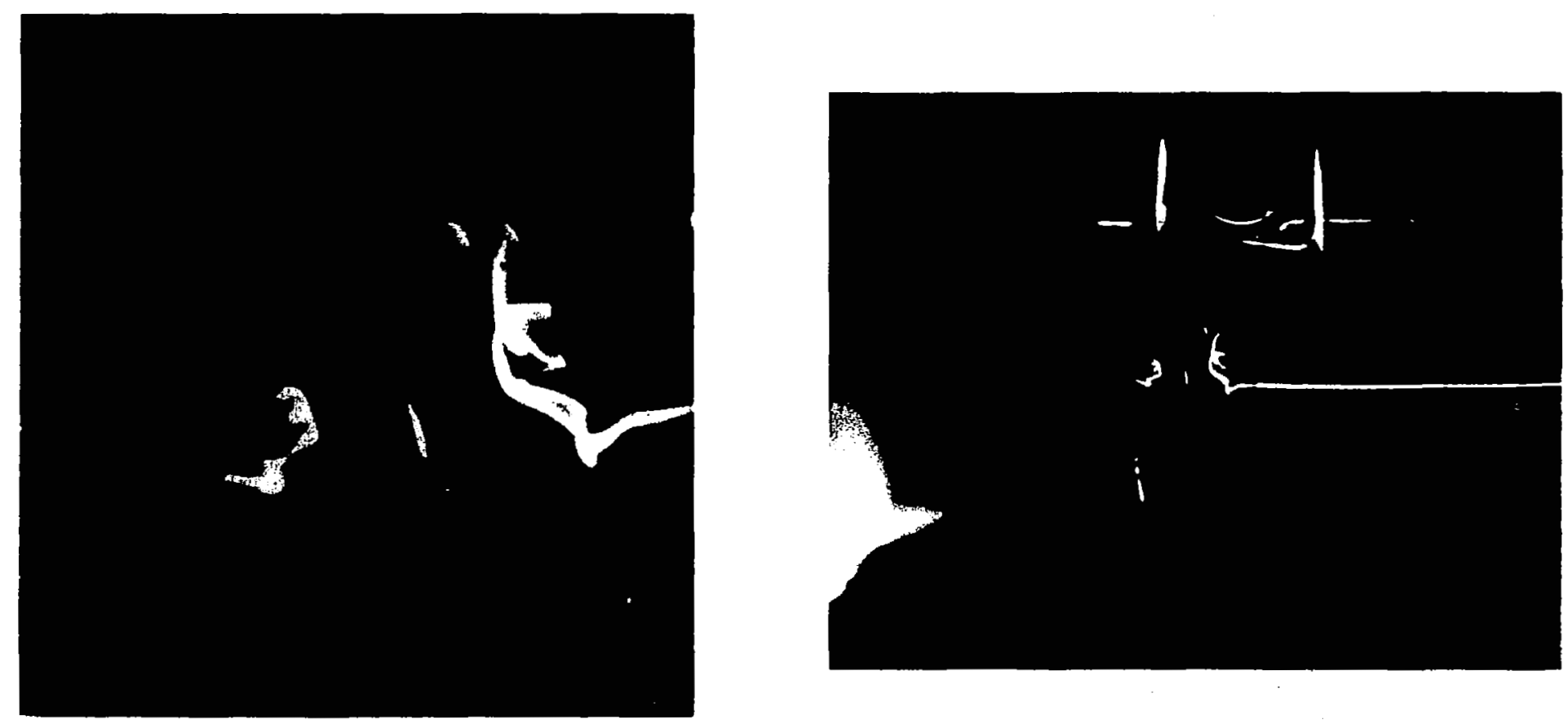

Enlargement

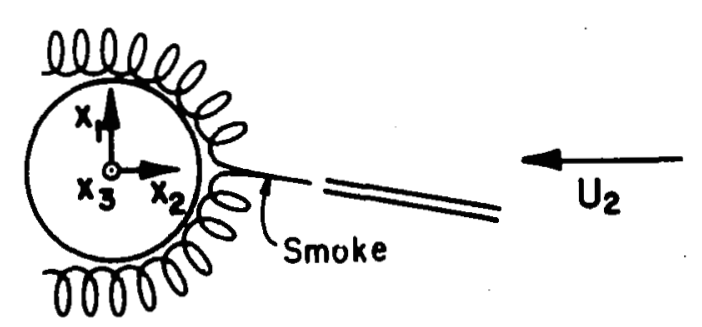

Fig. 8. Vortex flow pattern near the stagnation zone viewed in $x_{1} x_{2}$-plane (the stream plane); $\mathrm{f} / 2.8,1 / 60 \mathrm{~s}$. 


\section{FRAME}

N-S
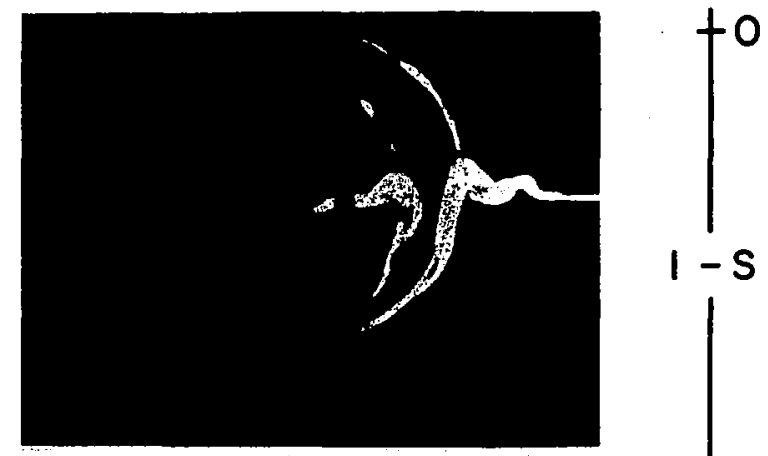

$1-S$
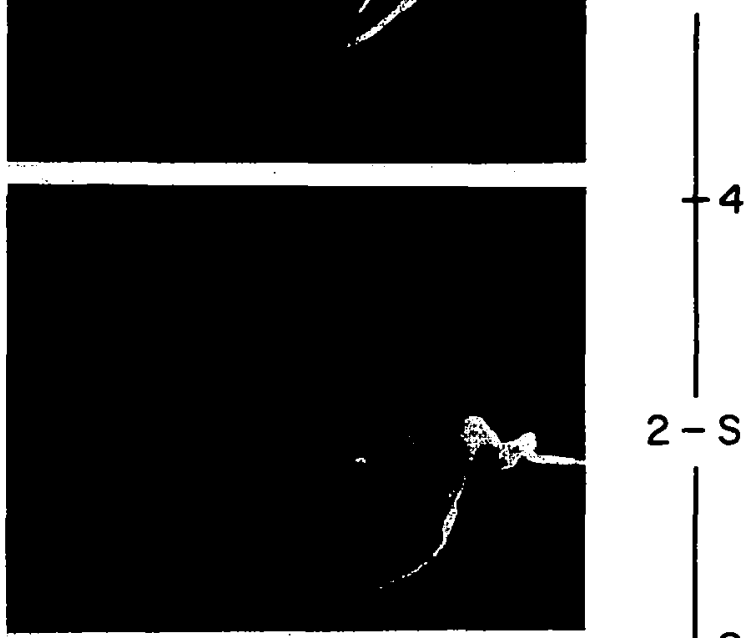

$-41 \frac{2}{3}$
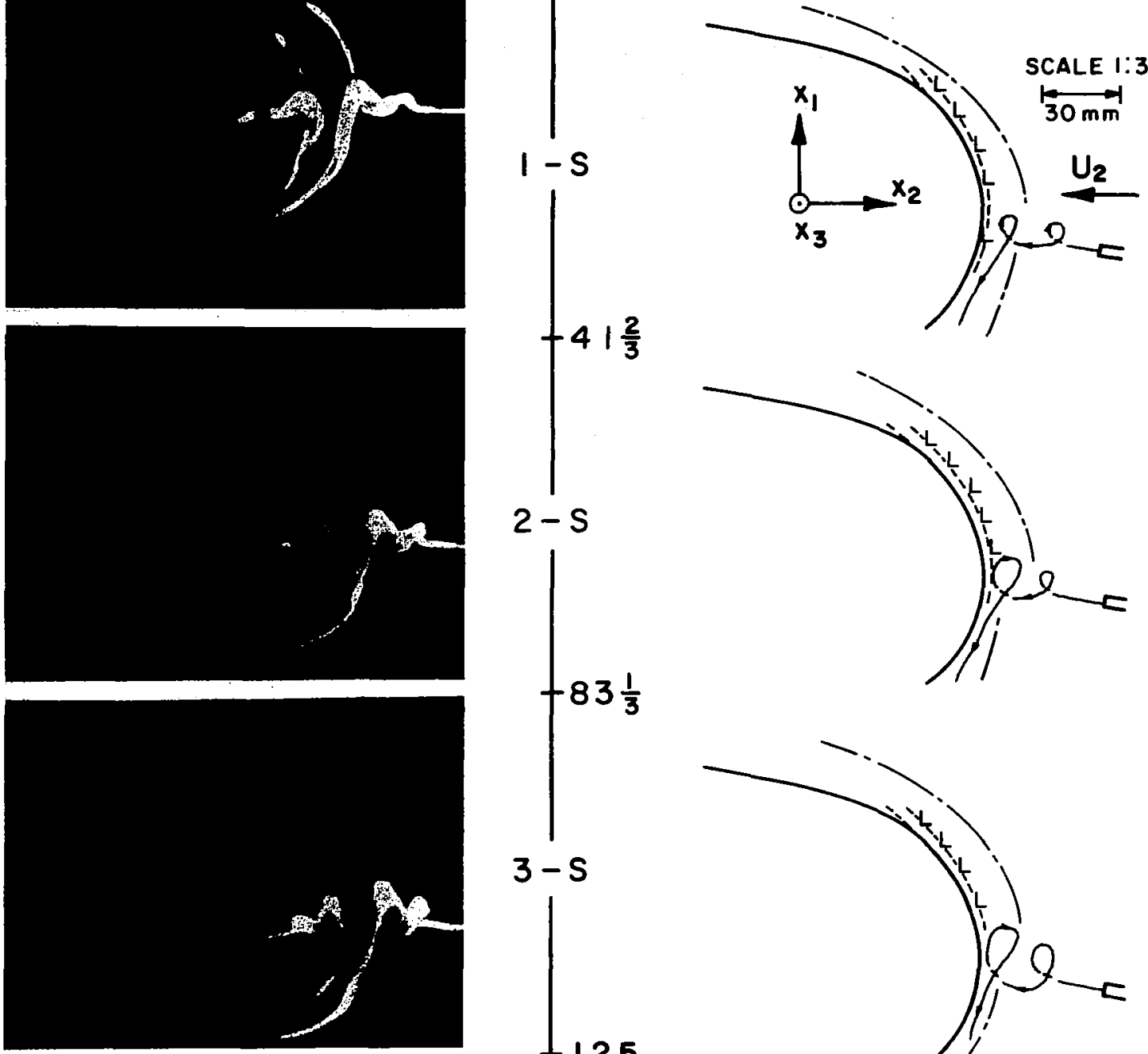

$-83 \frac{1}{3}$
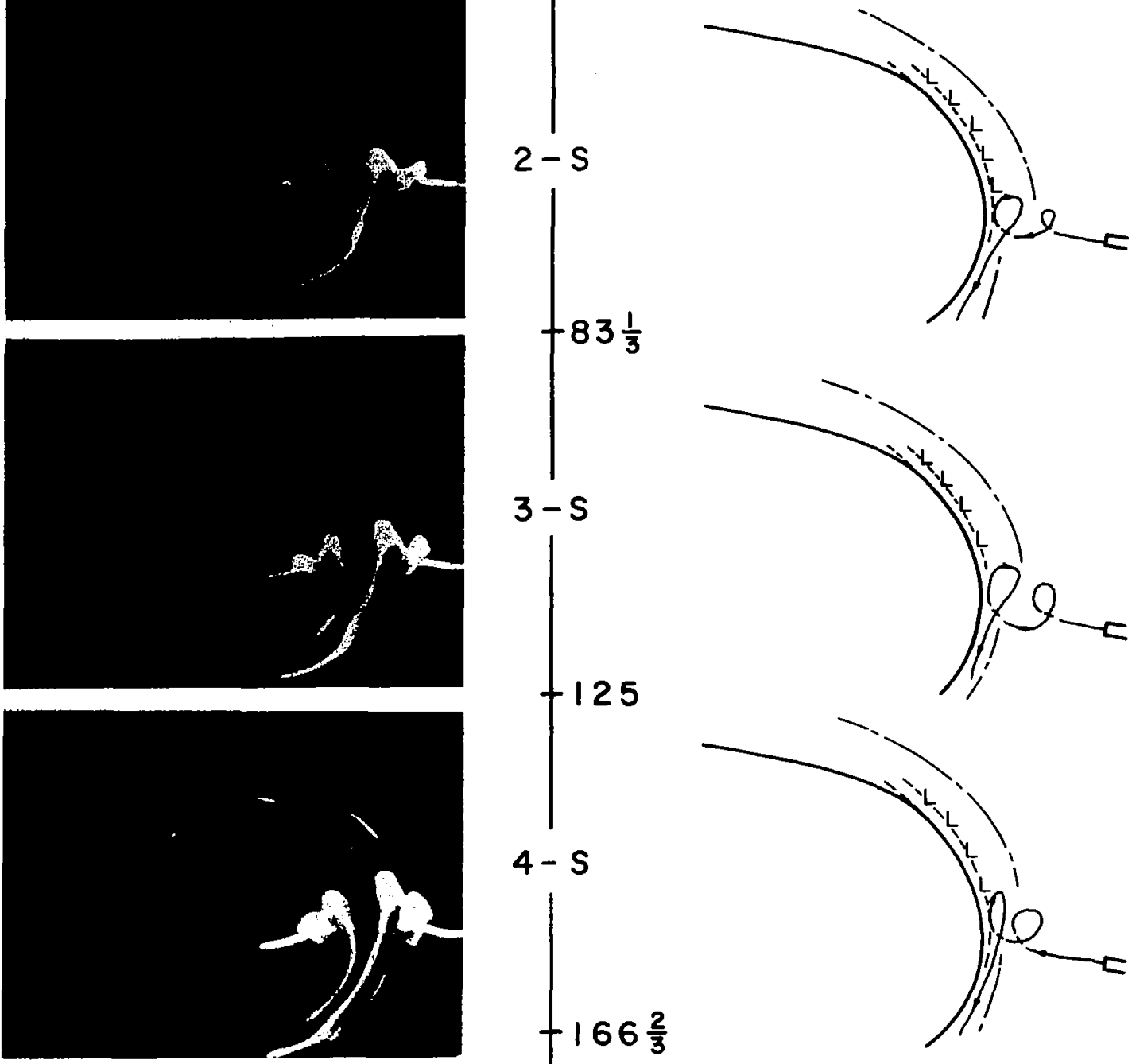

$2-s$

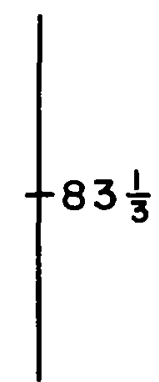

$3-s$

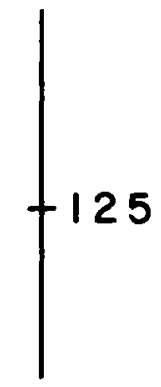

$4-S$
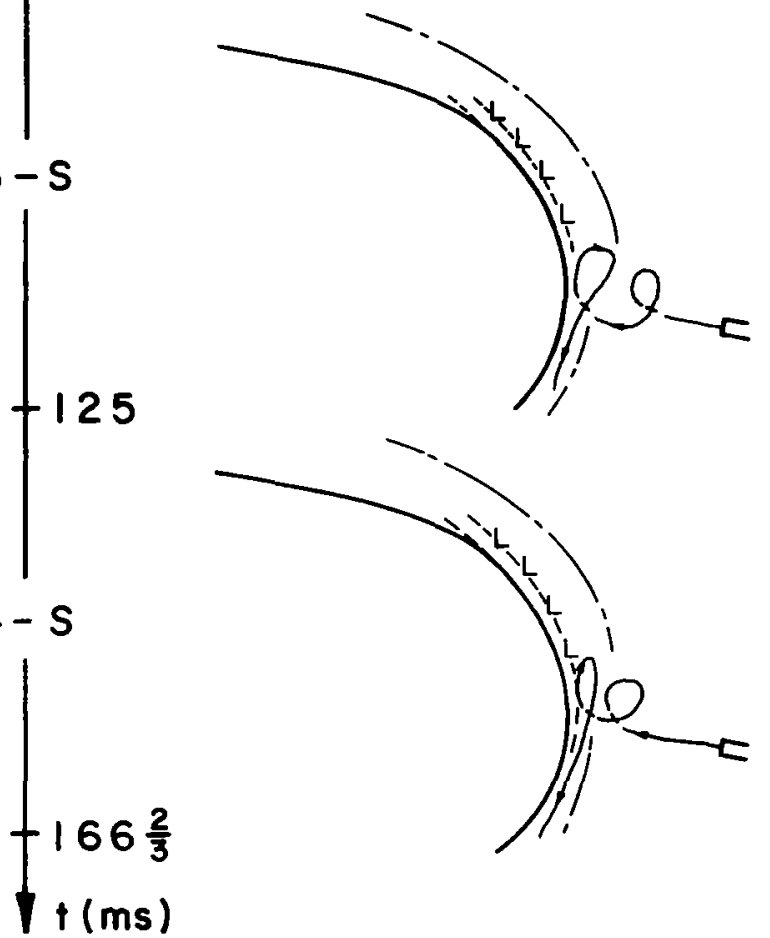

Fig. 9.1. Side-view sequence illustrating the time history of a tracked cross-vortex tube viewed in the $x_{1} x_{2}$-plane (the stream plane). 


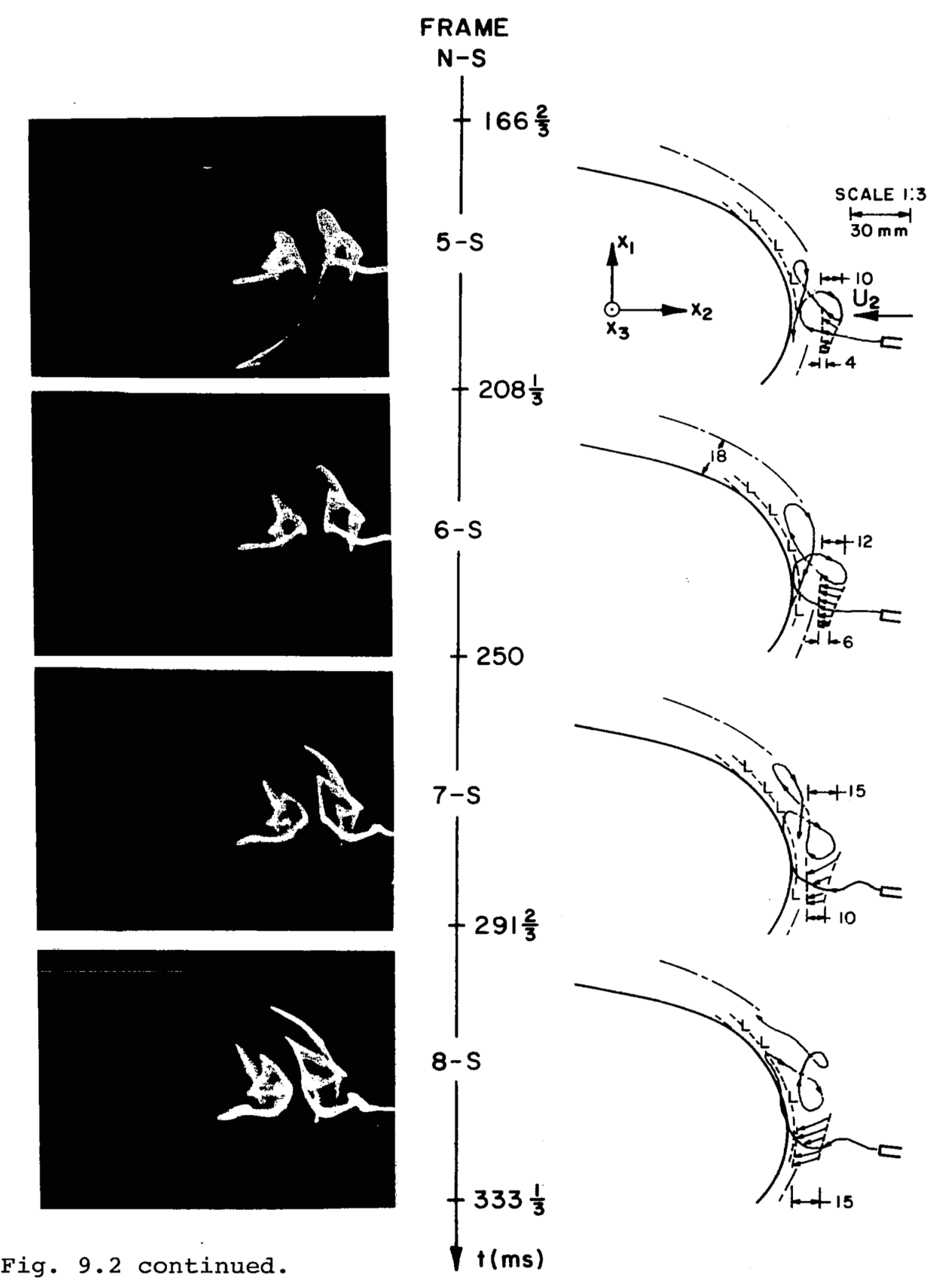




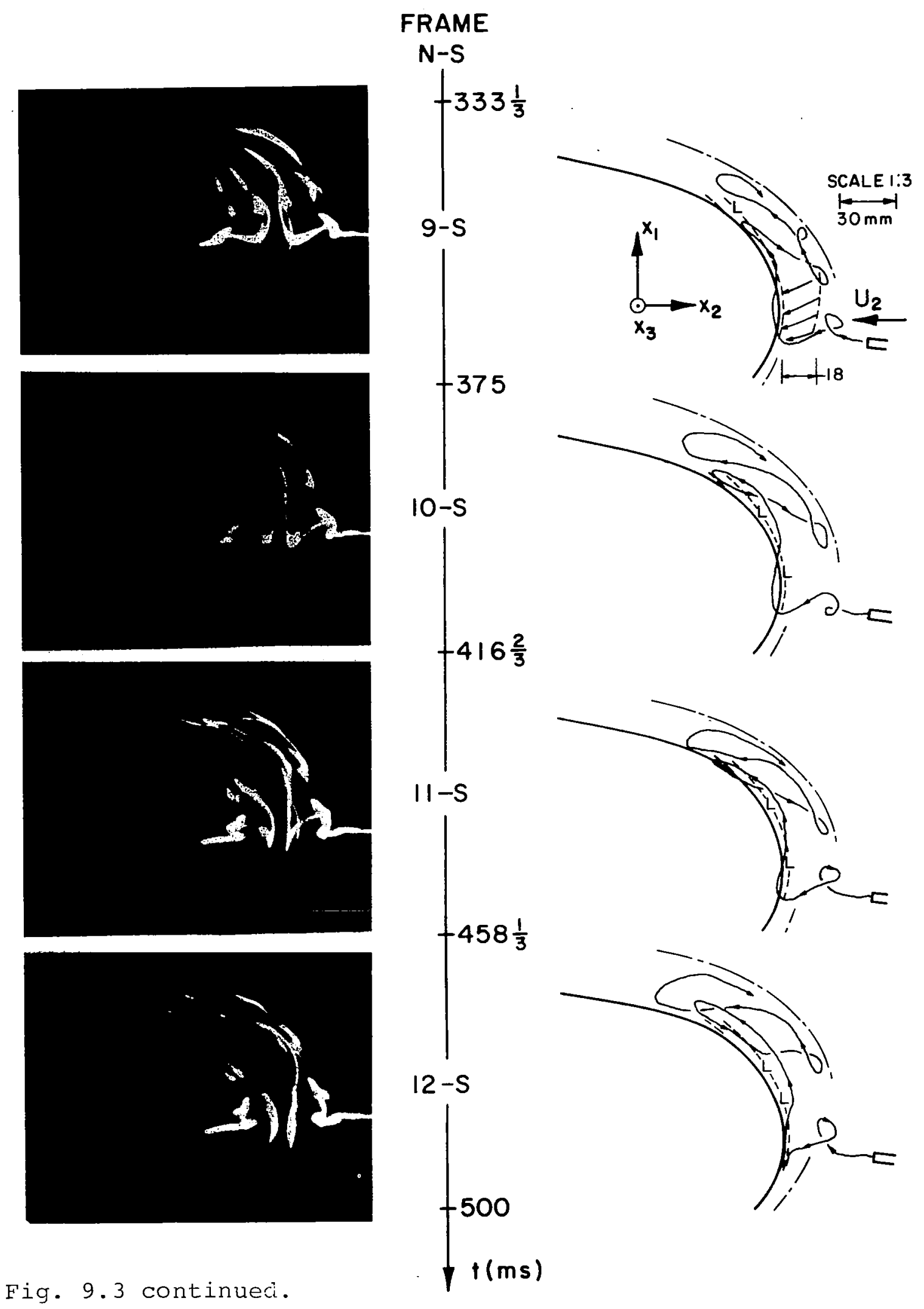




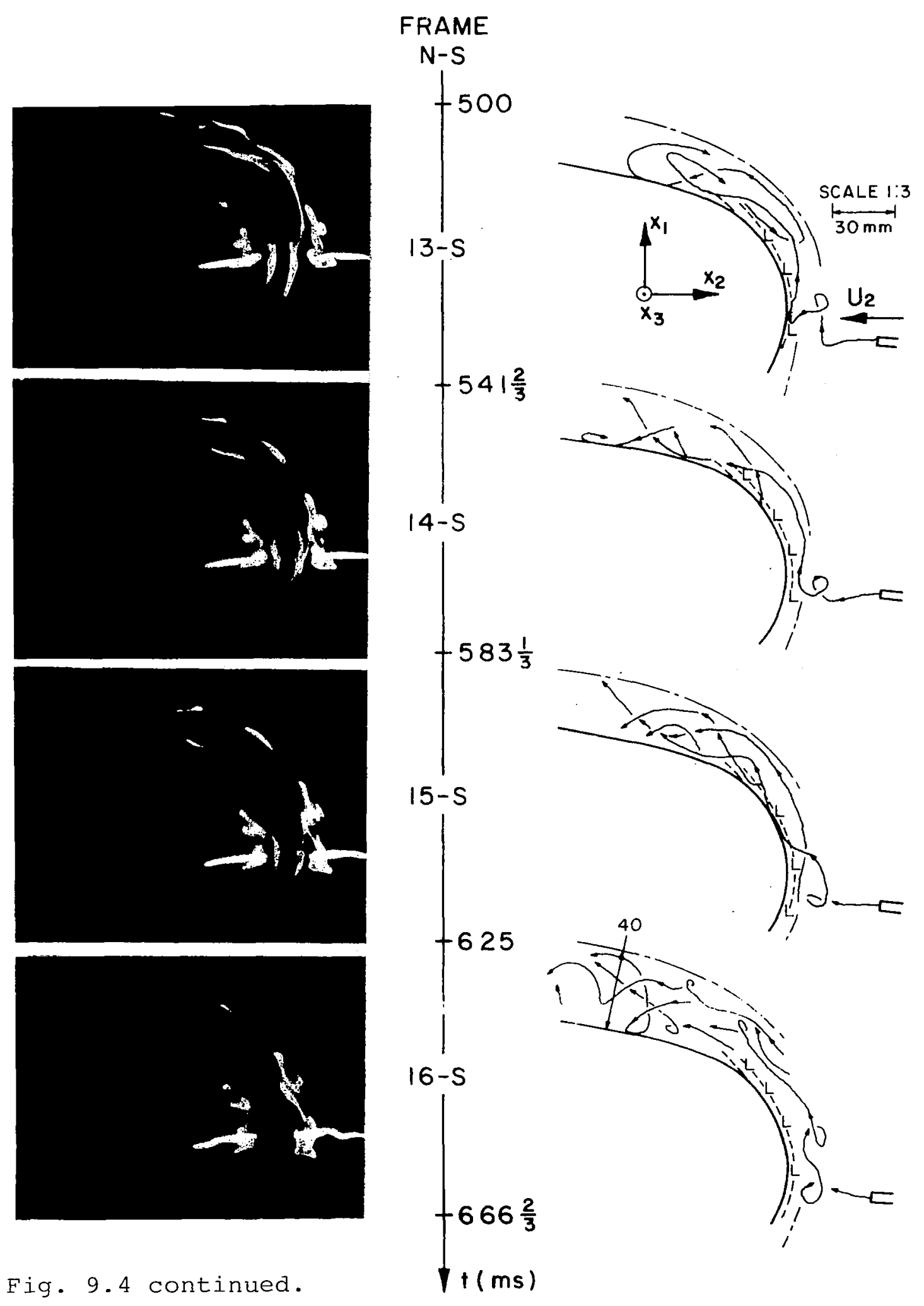




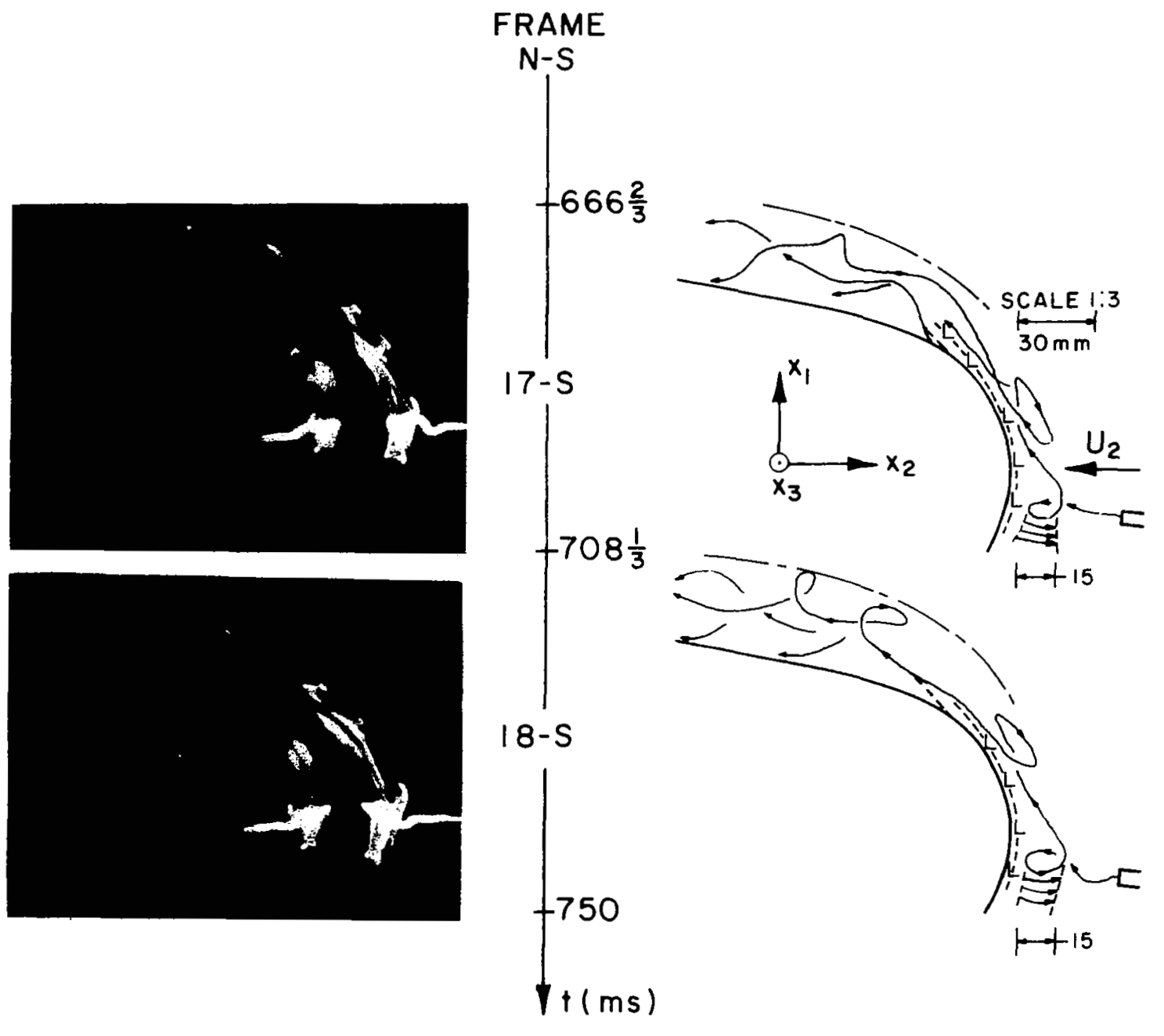

Pig. 9.5. continued. 

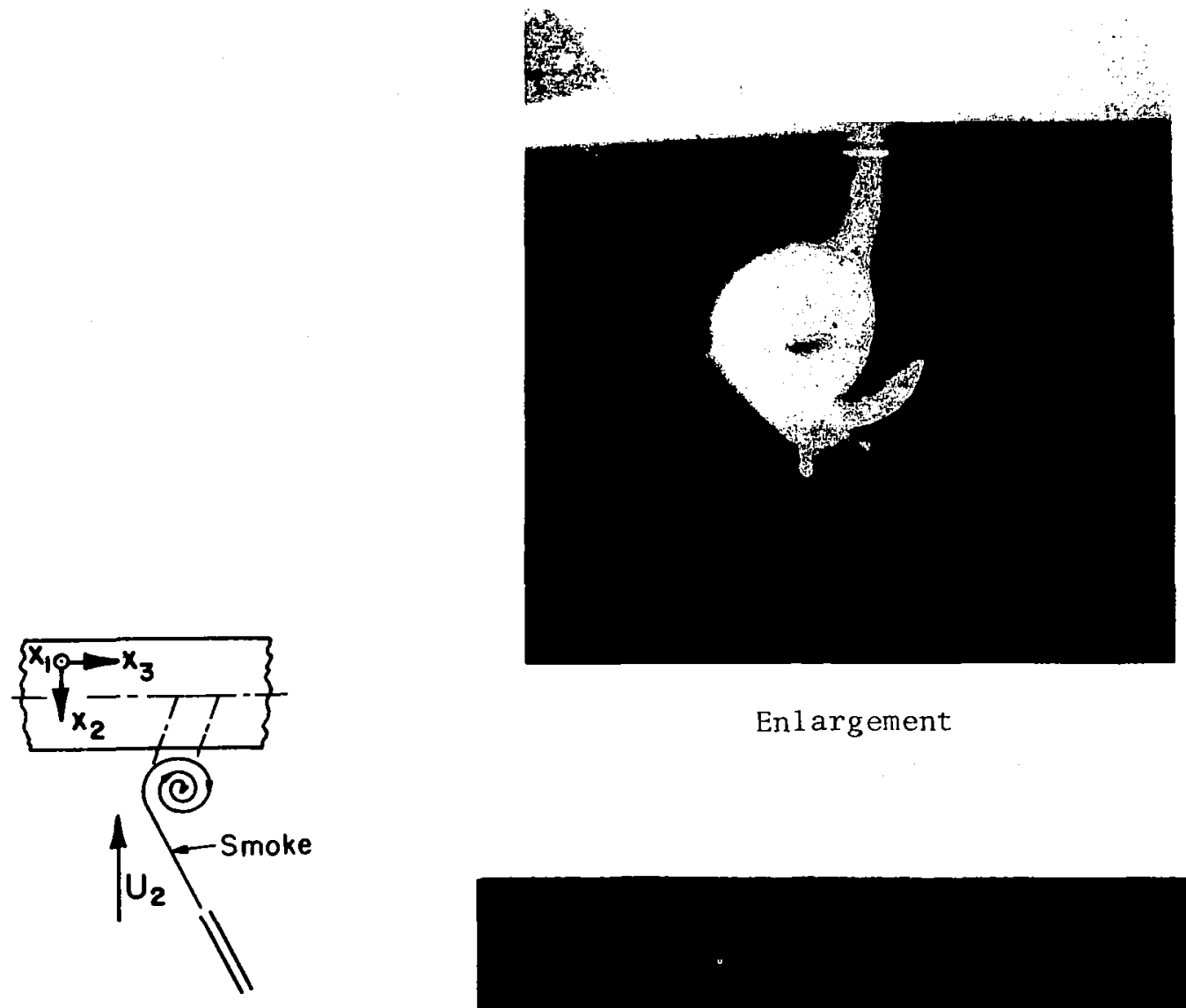

Enl argement

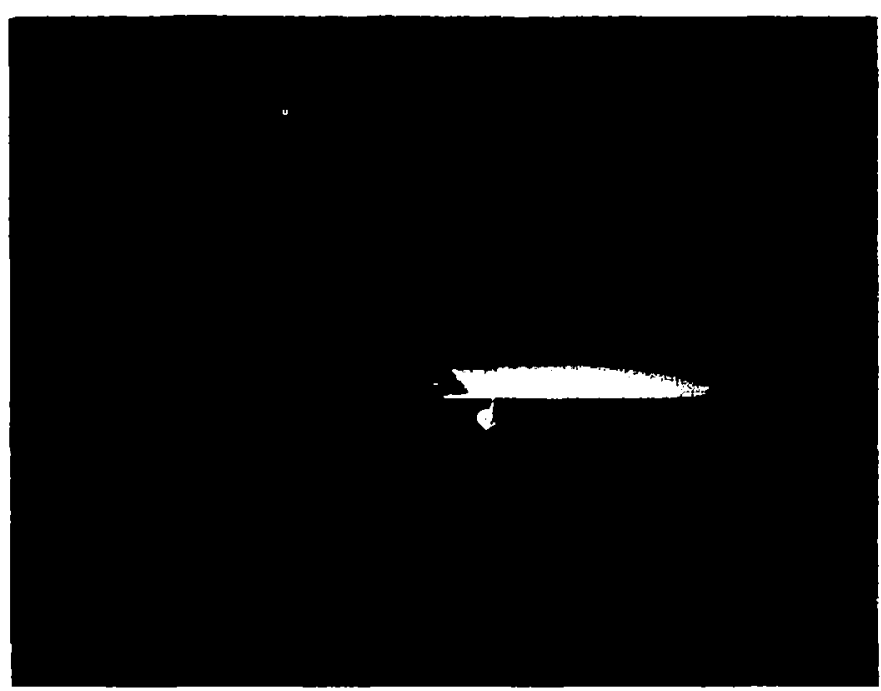

Fig. 10. Vortex flow pattern near the stagnation zone viewed in the $\mathrm{x}_{2} \mathrm{x}_{3}$-plane (the normal plane); $f / 4.5,1 / 60 \mathrm{~s}$. 

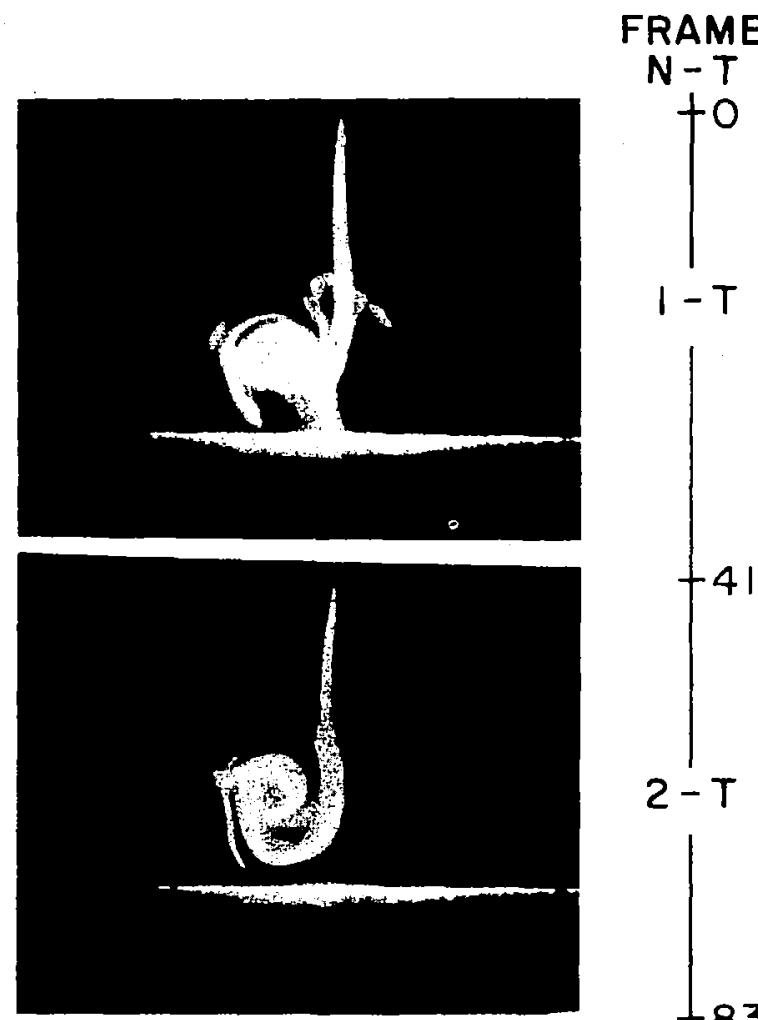

$1-\mathrm{T}$
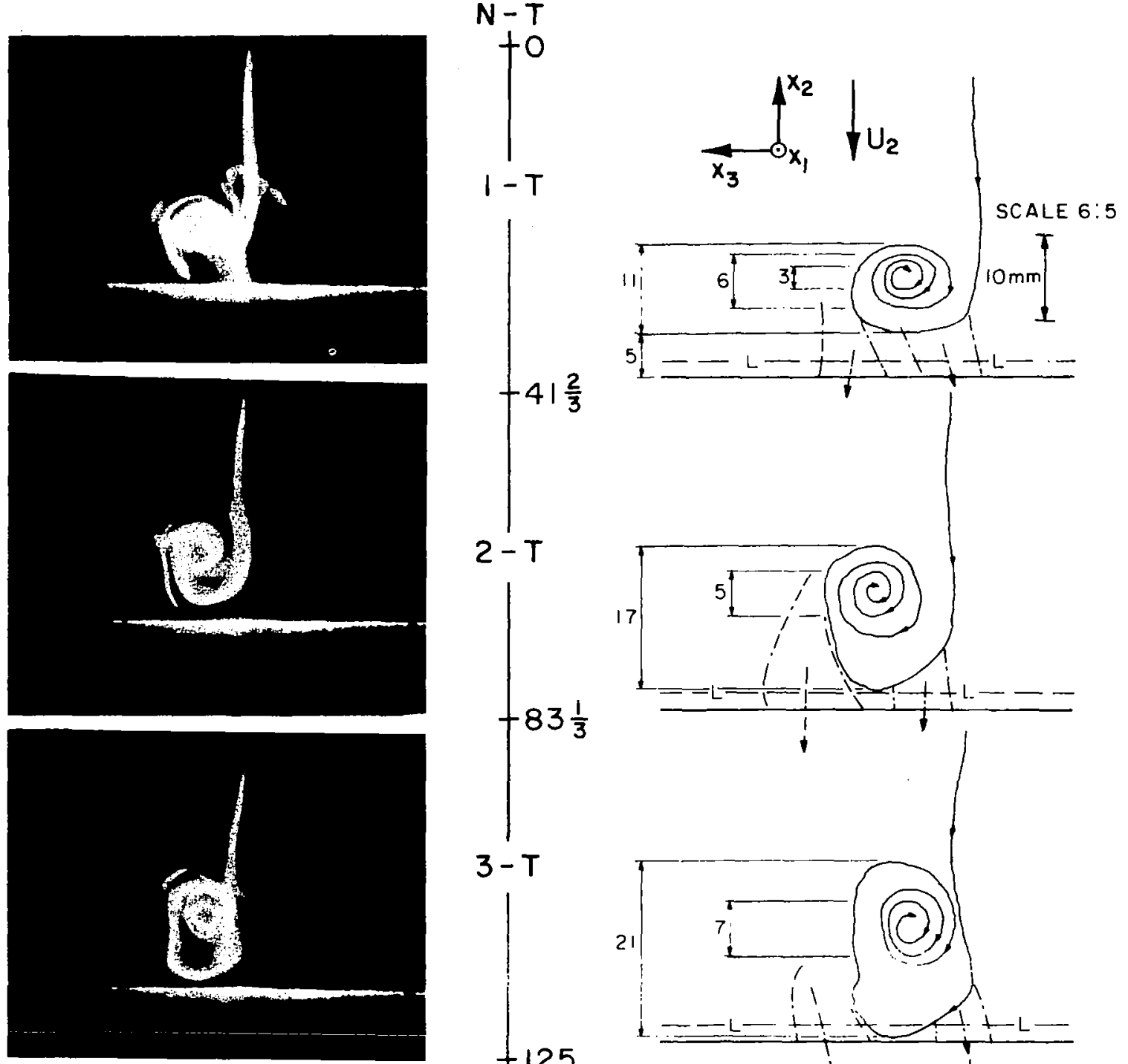

$2-\mathrm{T}$
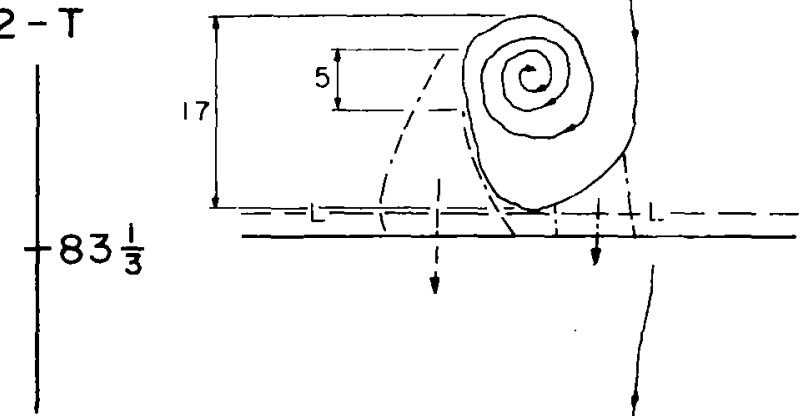

$3-\mathrm{T}$
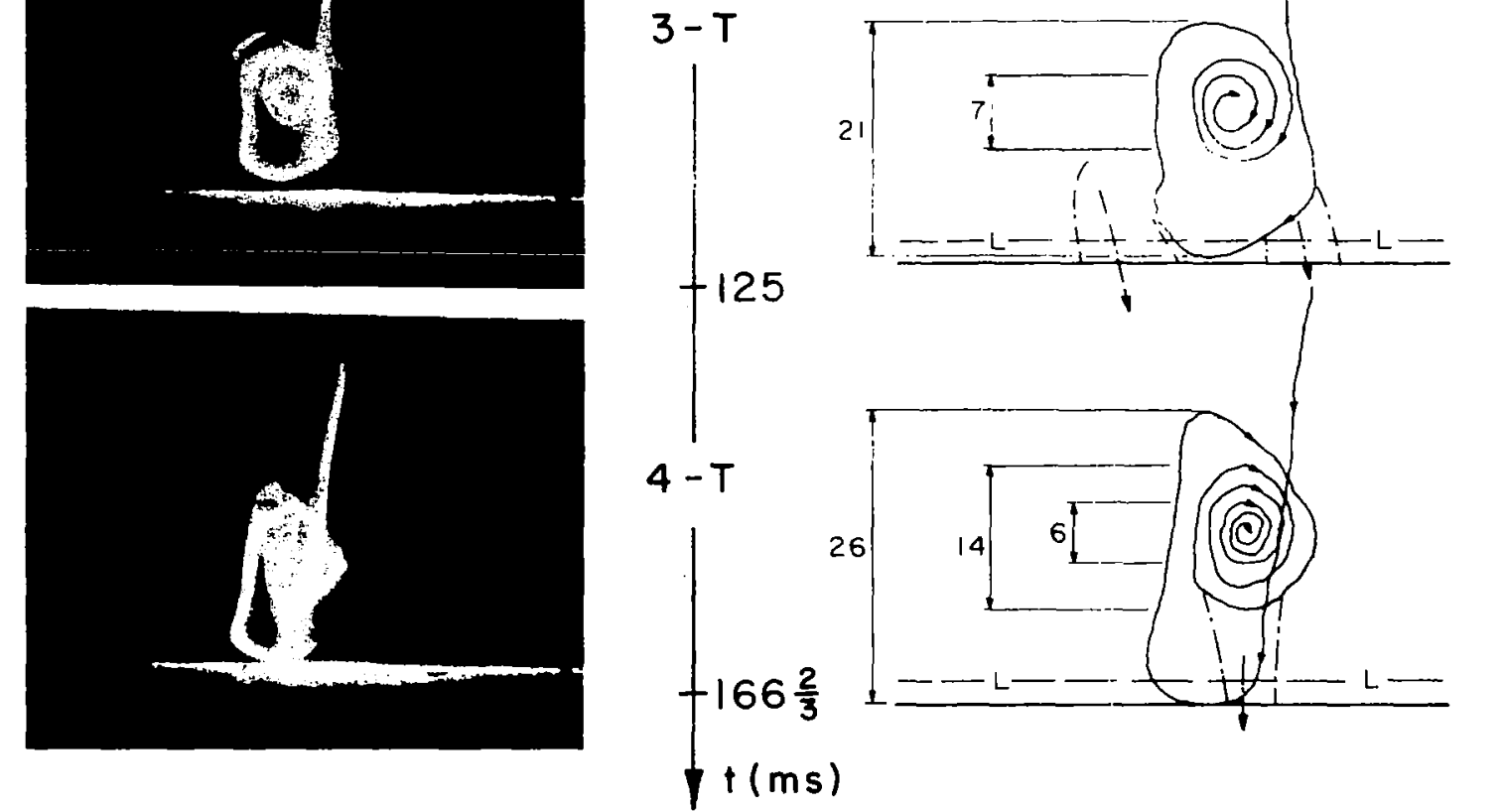

Fig. Il.1. Top-view sequence illustrating the evolution of a standing cross-vortex tube near the stagnation zone viewed in the $x_{2} x_{3}-p$ lane (the normal plane). 


\section{FRAME \\ $N-T$}
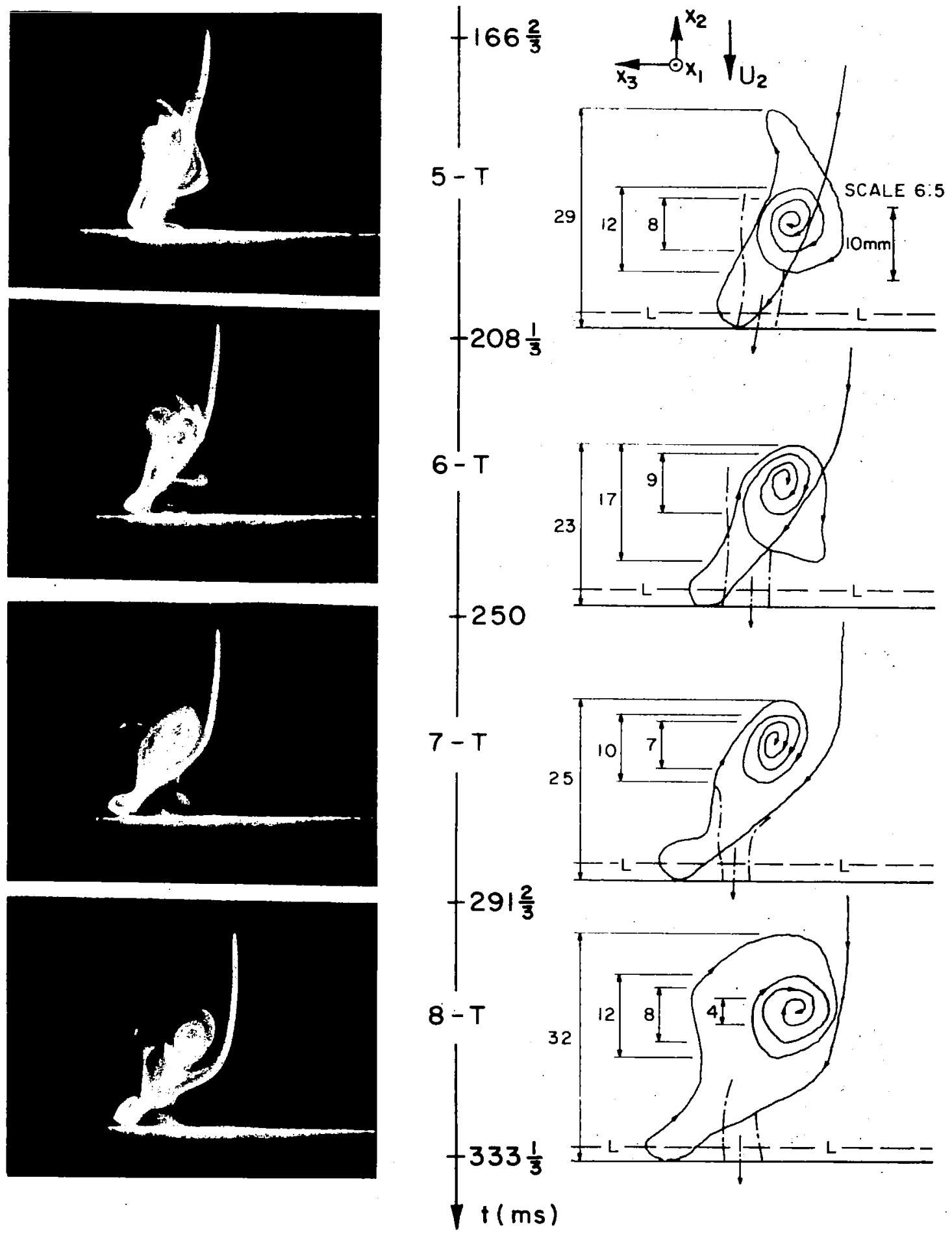

Fig. 11.2. continued. 


\section{FRAME}
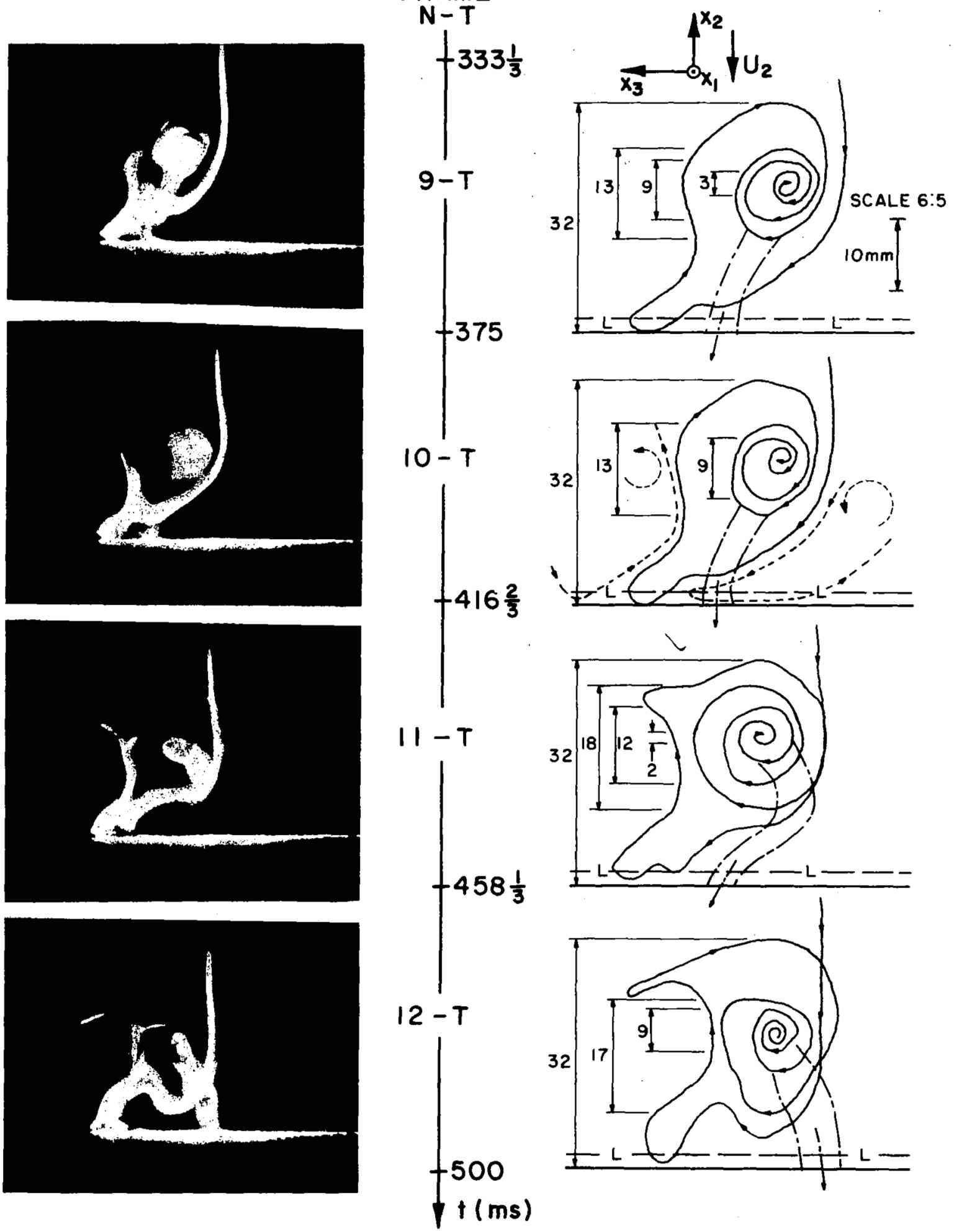

Fig. 11.3. continued. 


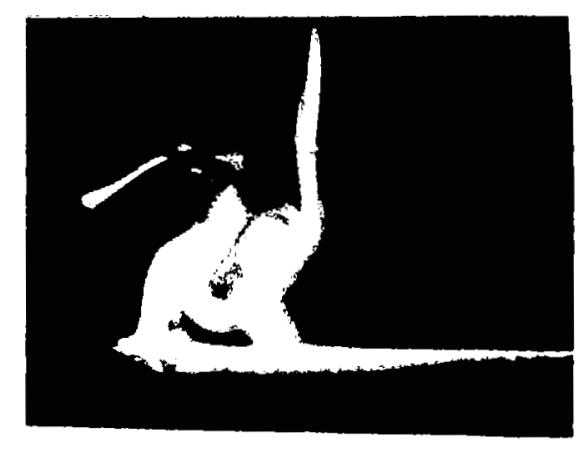

FRAME
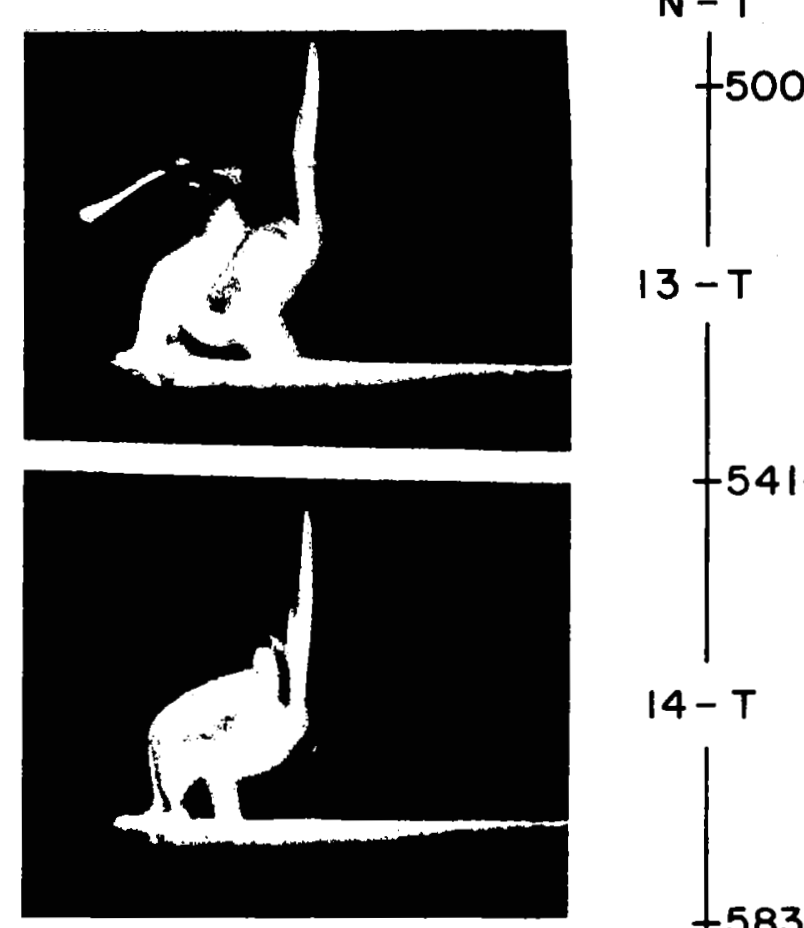

$13-T$
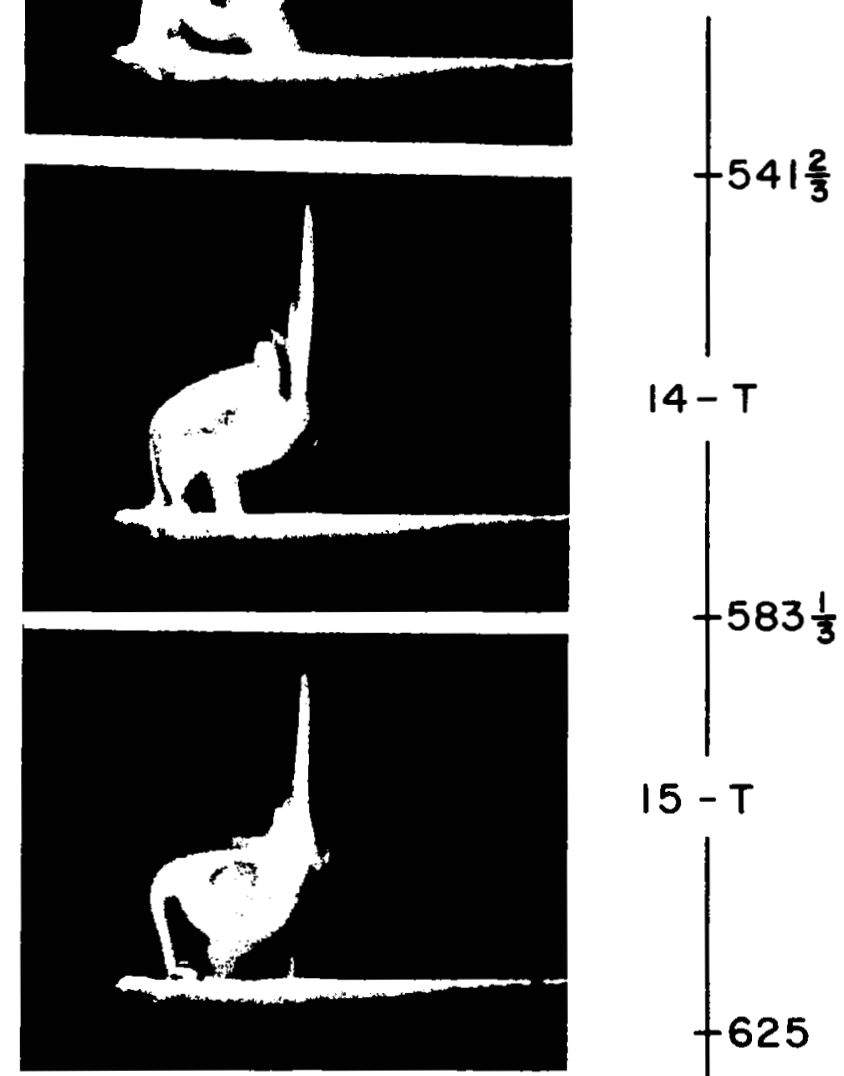

14-T
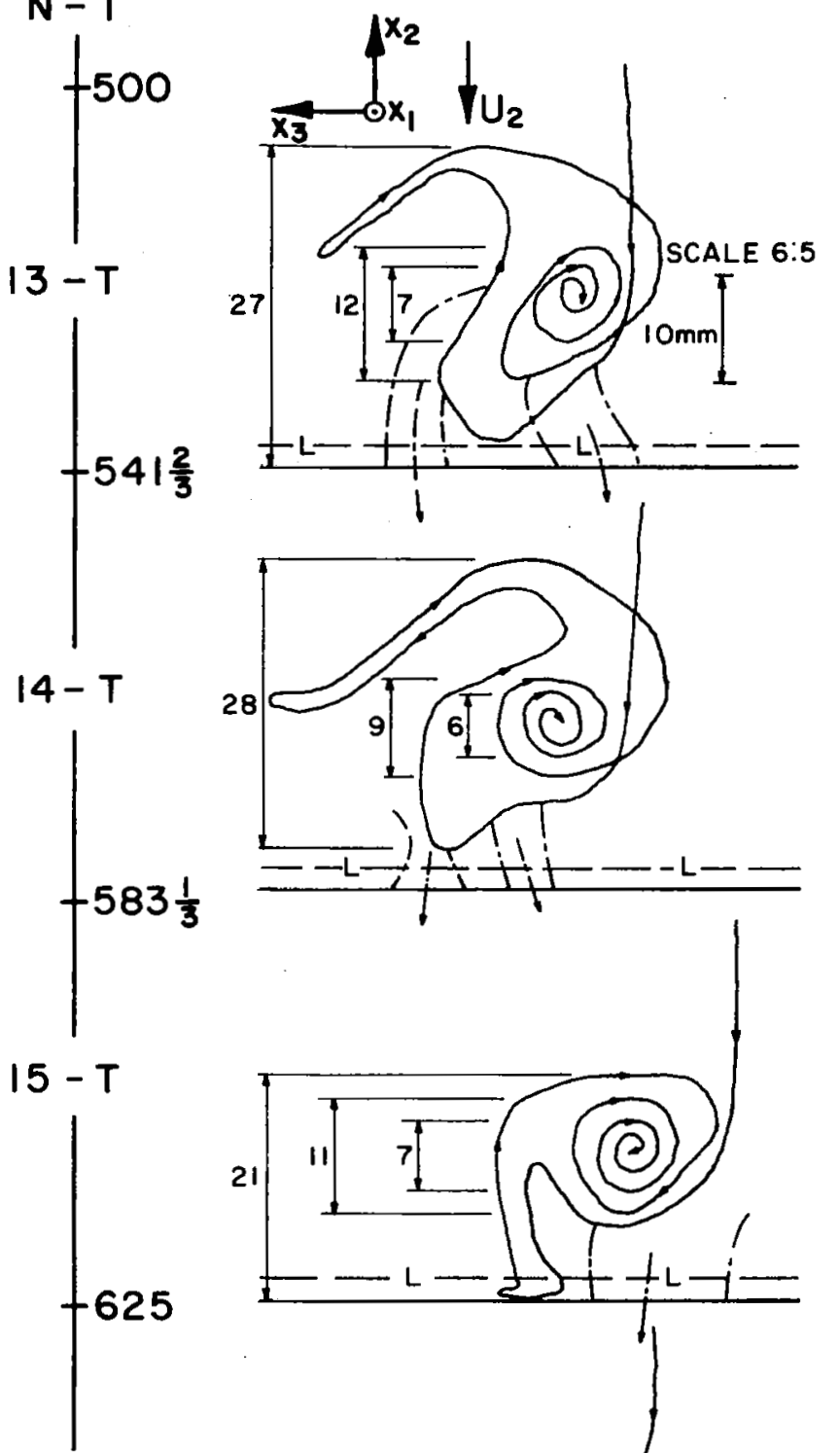

$16-T$
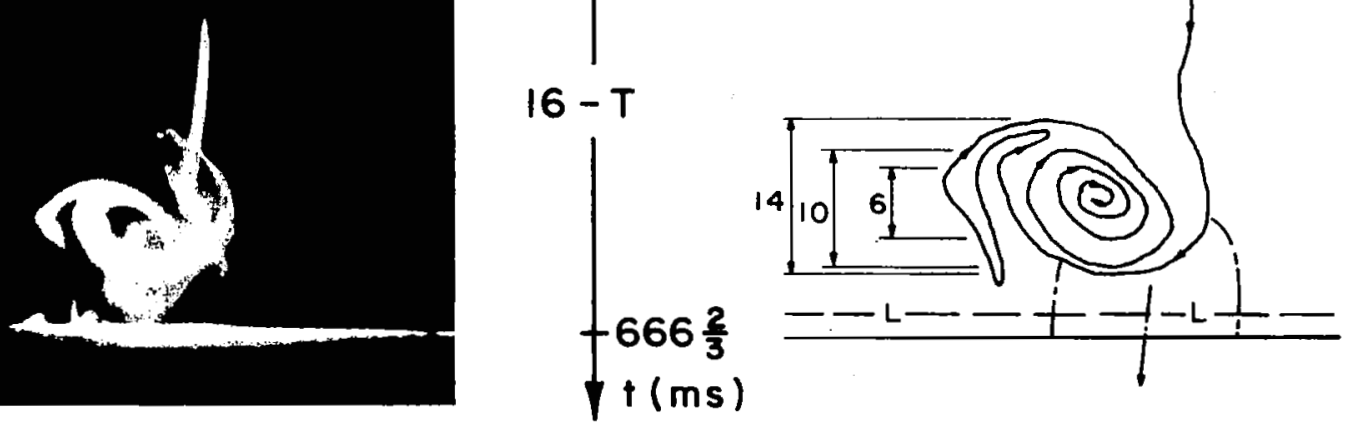

Fig. 11.4. continued. 

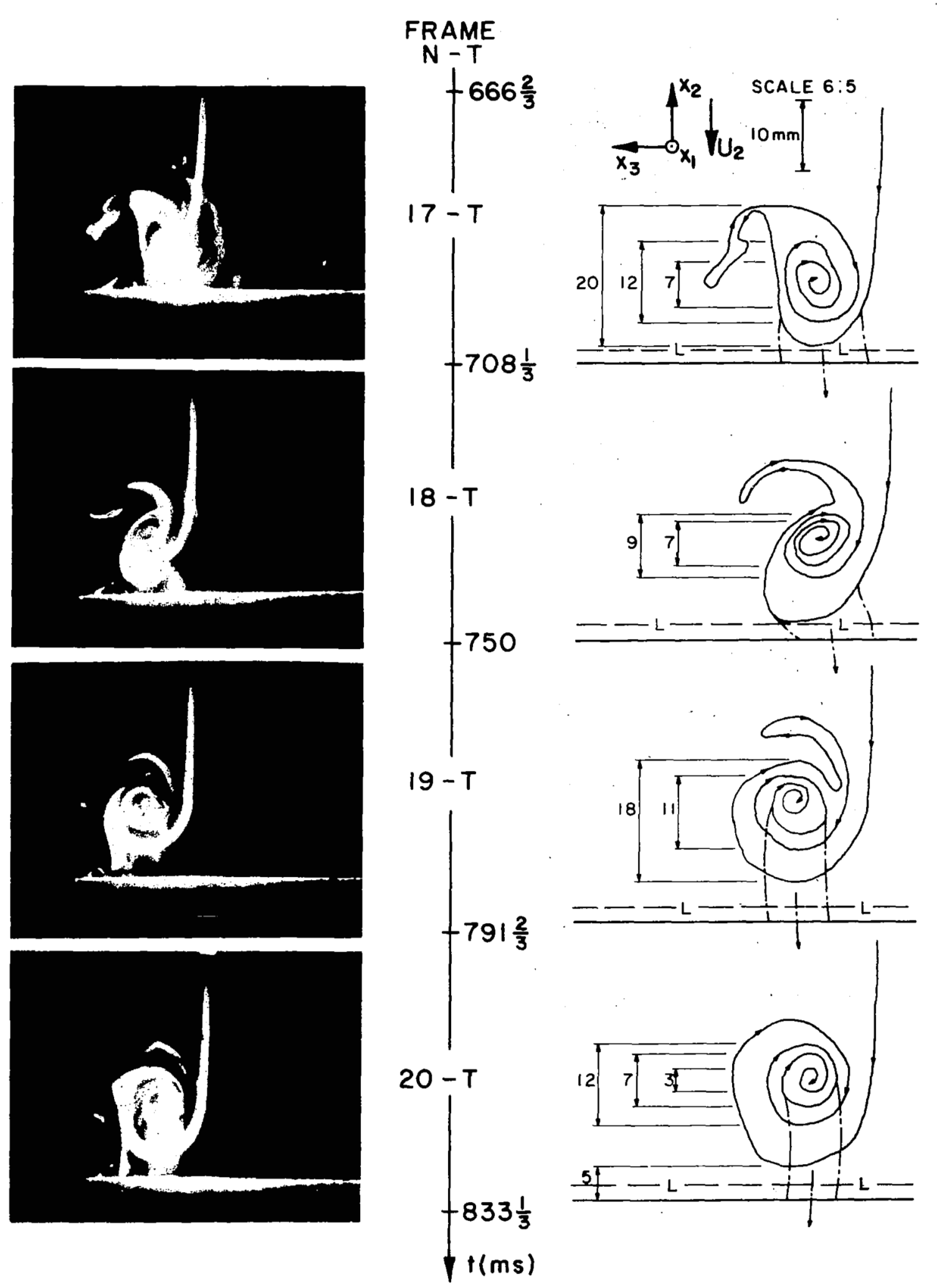

Fig. 11.5. continued. 


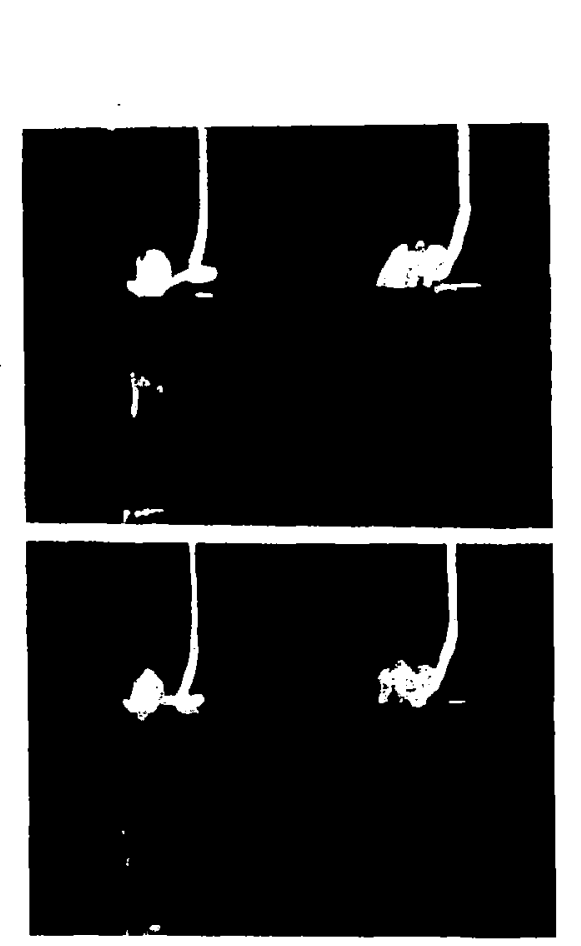

FRAME

$N-T / D$

$\int_{x_{3}}^{x_{2}} \overbrace{x_{1}} t_{u_{2}}^{\text {SCALE ::4 }}$
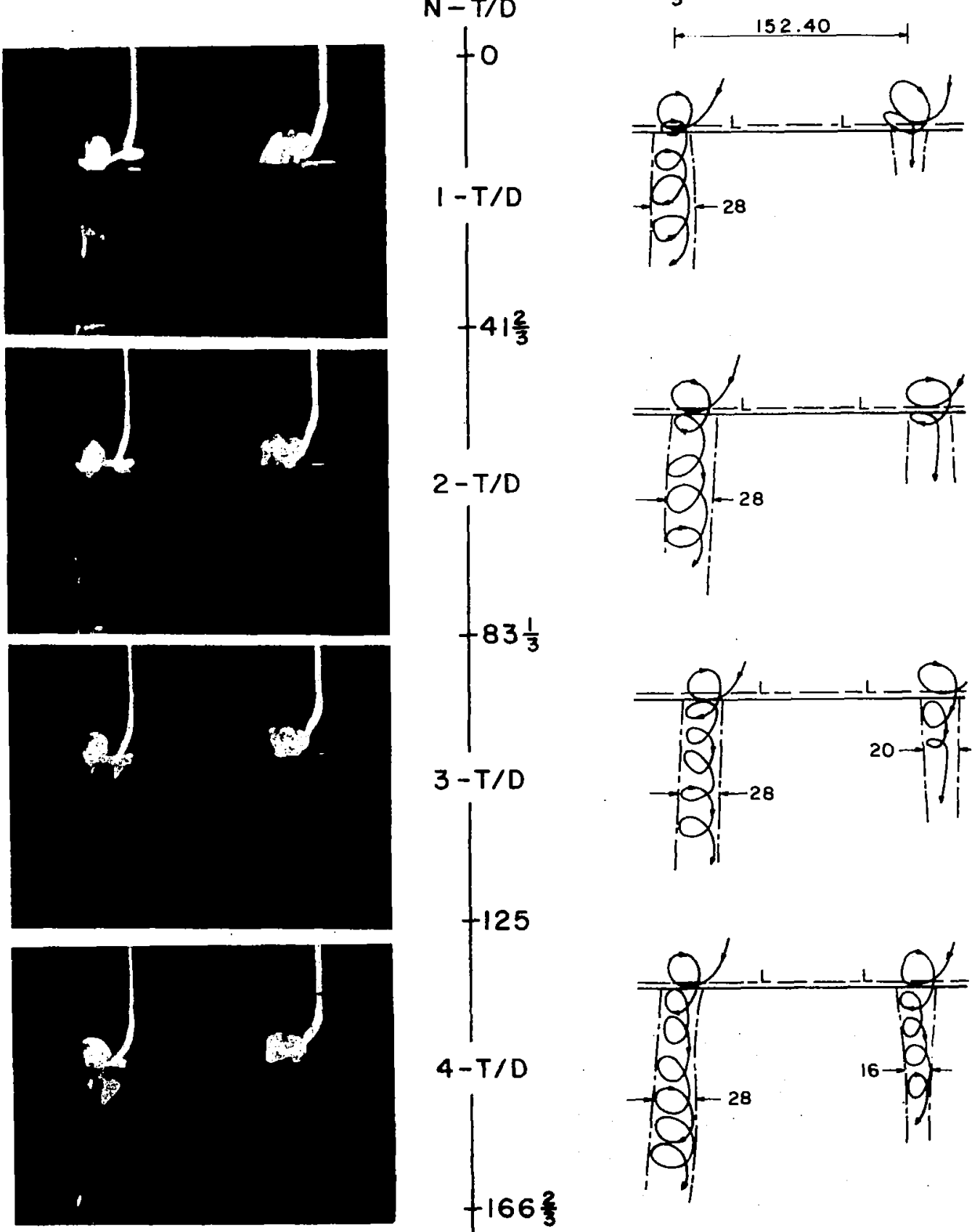

$-125$

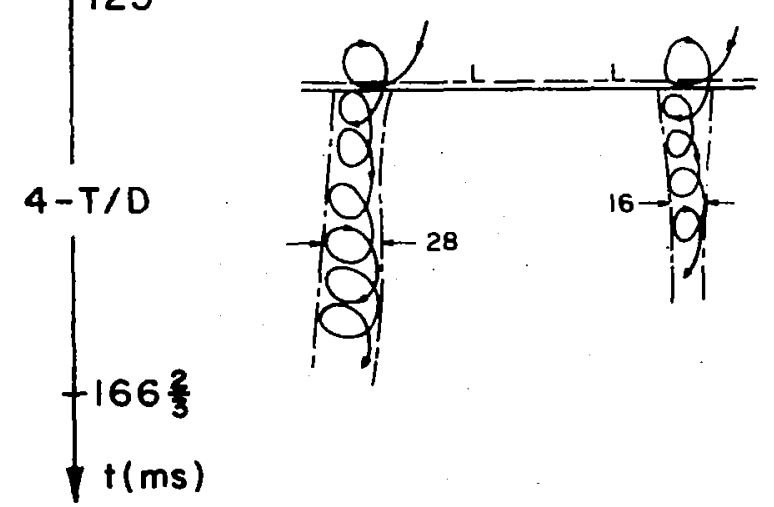

Fig. 12.1. Top-view sequence illustrating the coherent vortex flow pattern along the stagnation-zone span and the winding of the stretched cross-vortex tubes over the cylinder viewed in the $\mathrm{x}_{2} \mathrm{x}_{3}$-plane (the normal plane). 


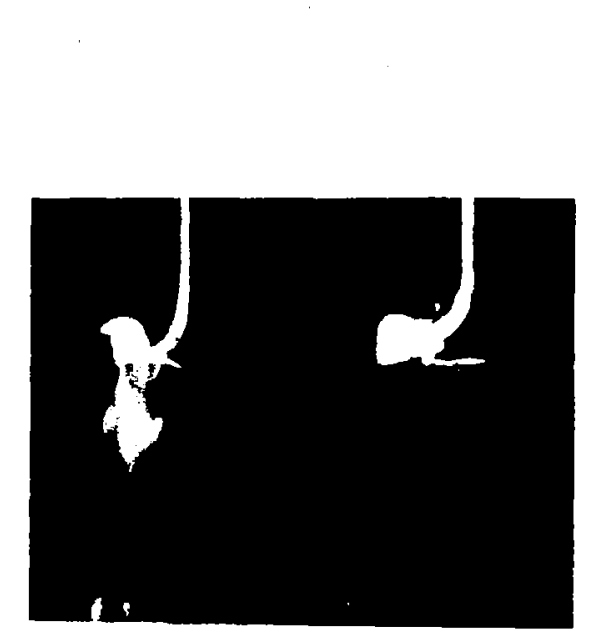

FRAME

$N-T / D$
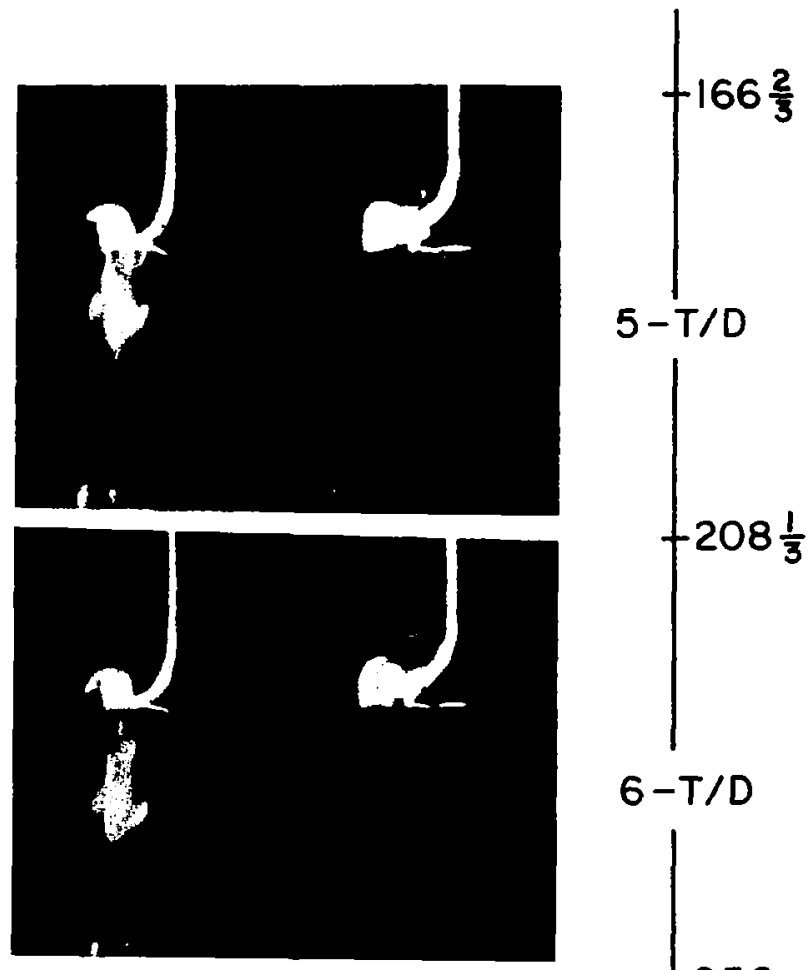

$6-T / D$
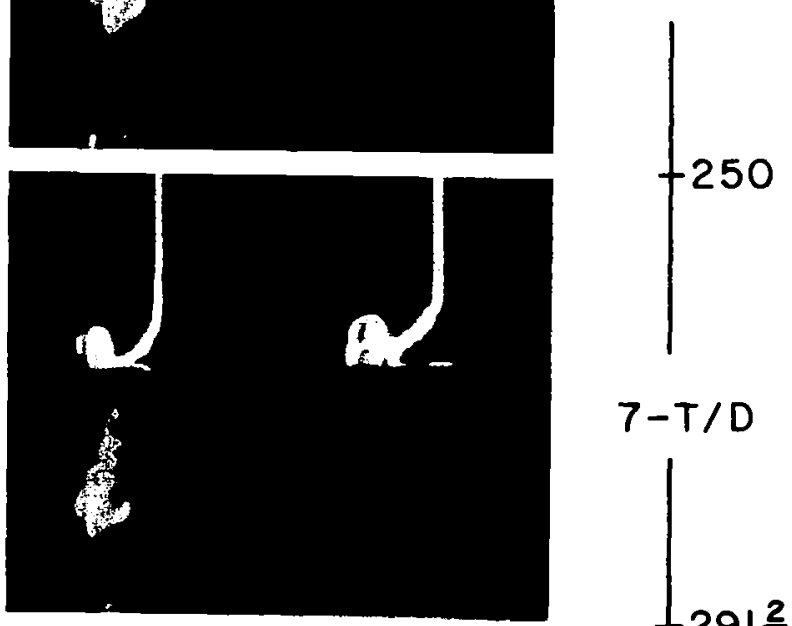

7-T/D
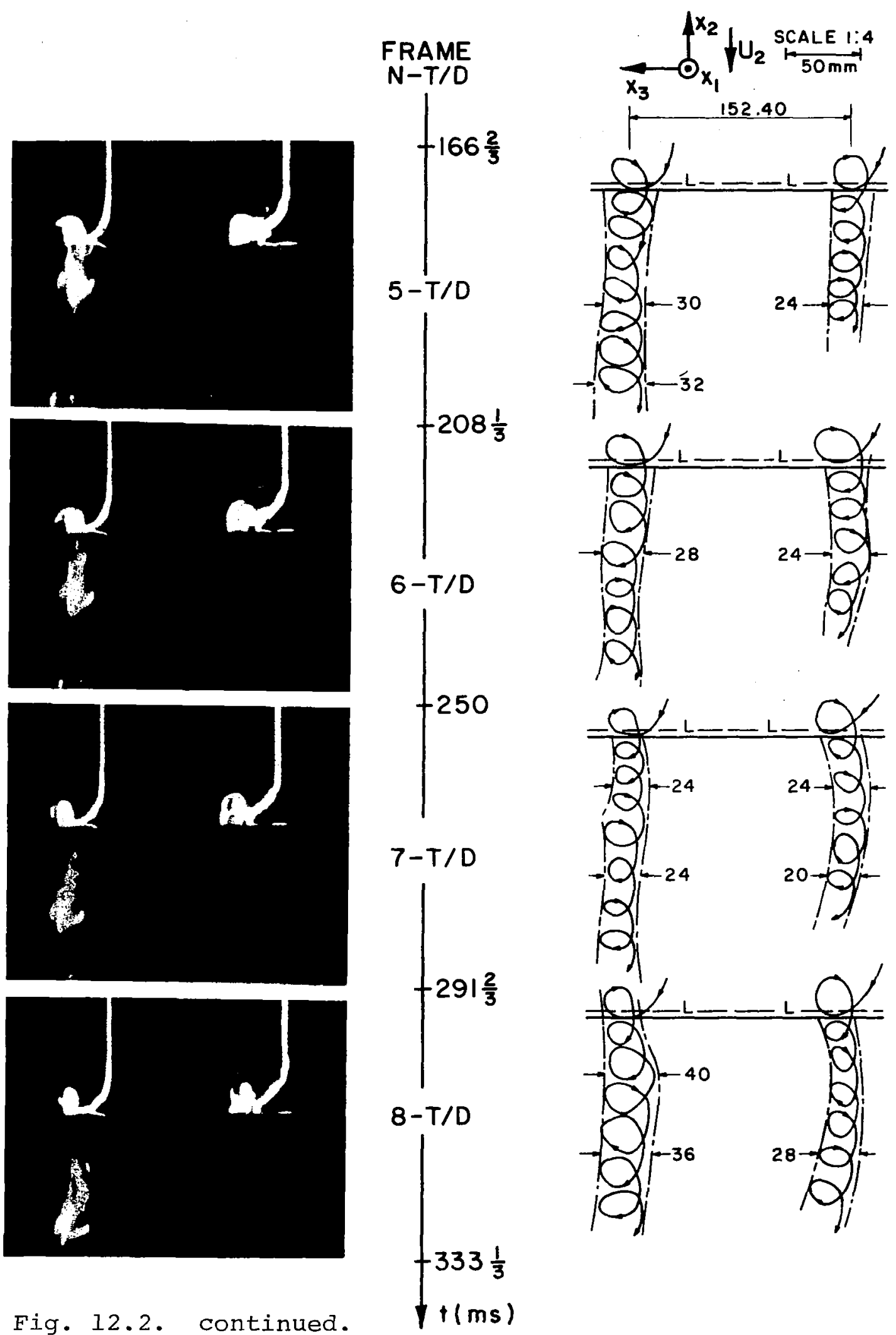




Fig. 13.1. Rear-view sequence illustrating the tilting of a stretched cross-vortex tube and the growth of a turbulent boundary layer viewed in the $x_{1} x_{3}$-plane (the cross plane). 


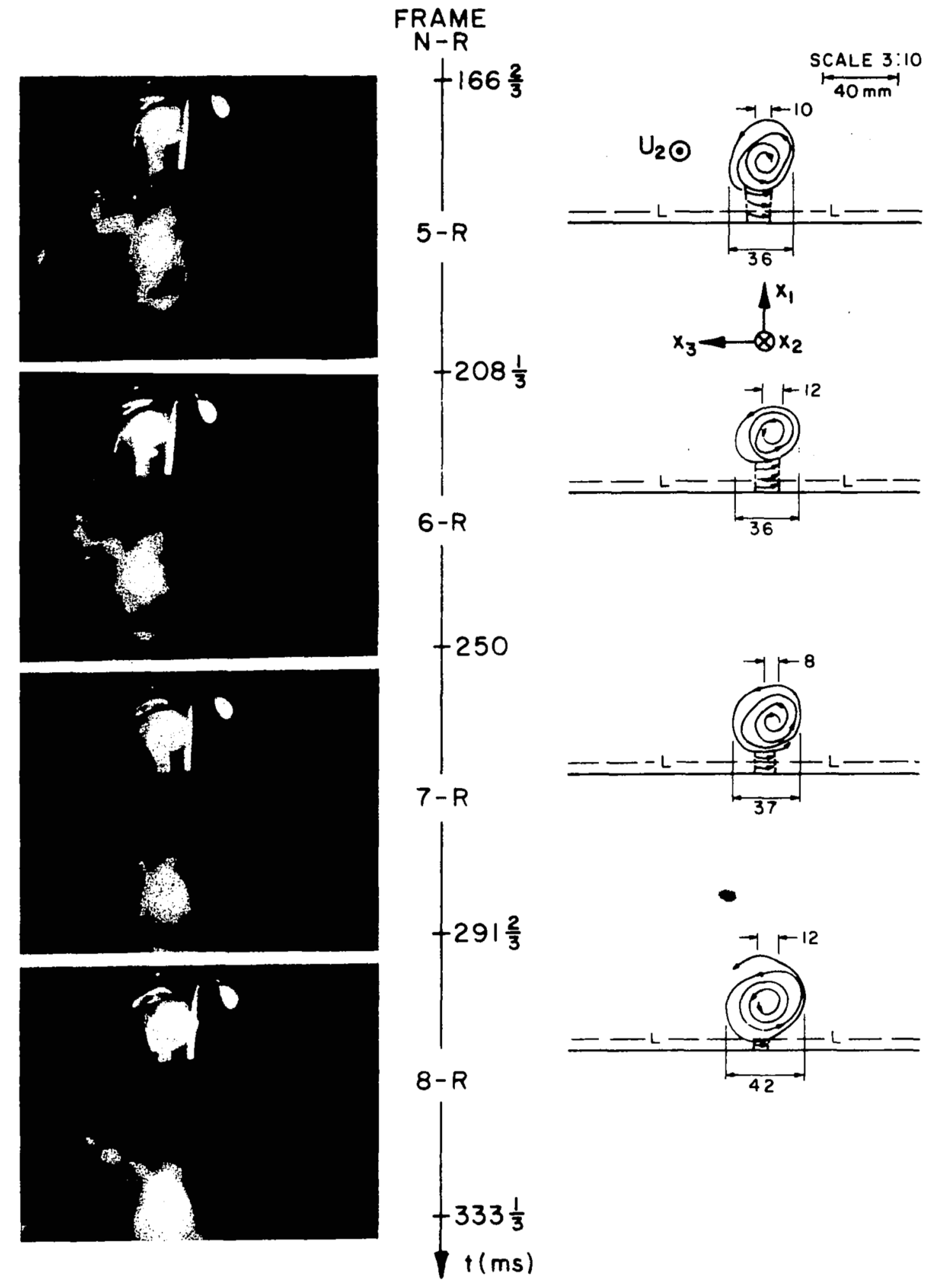

Fig. 13.2. continued. 


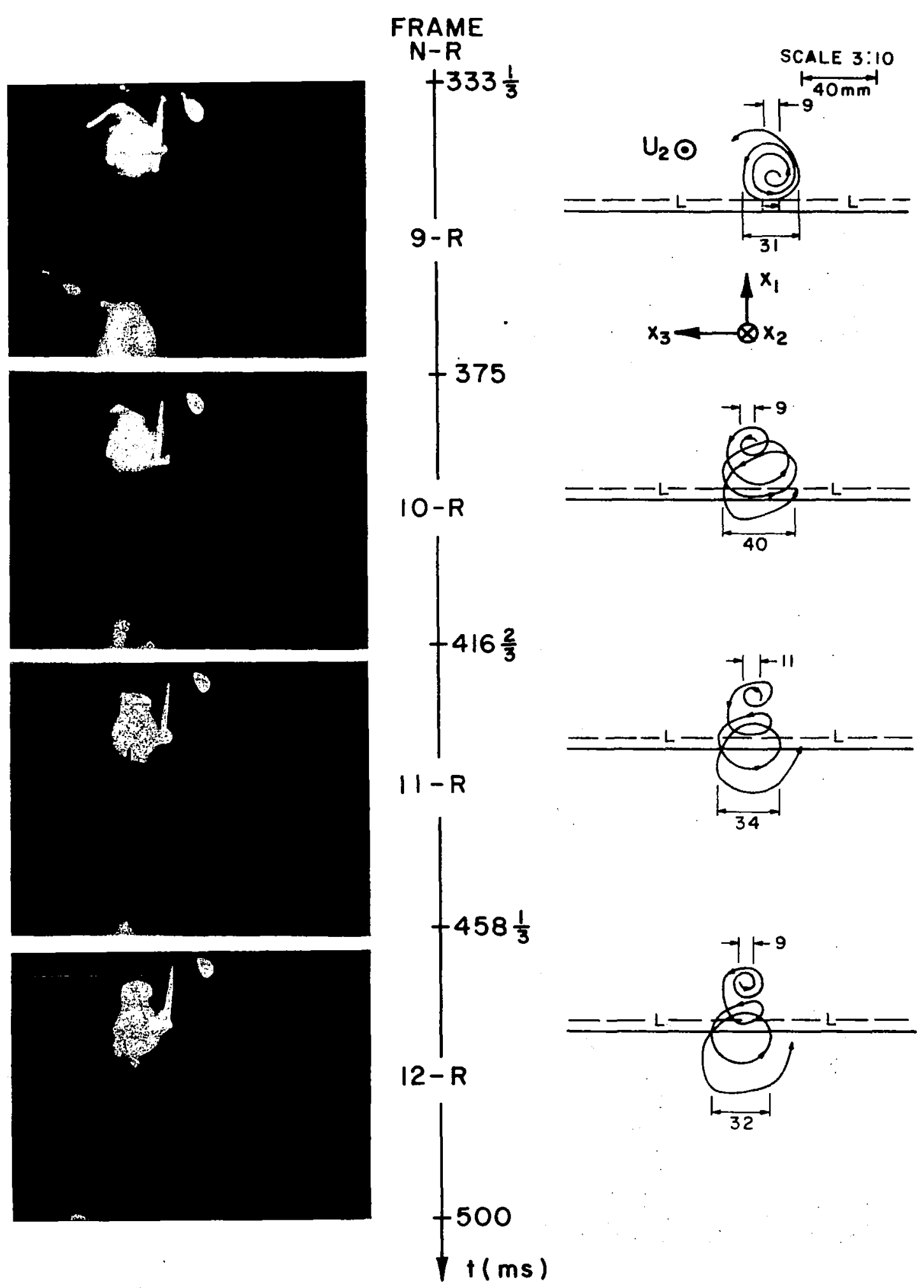

Fig. 13.3. continued. 


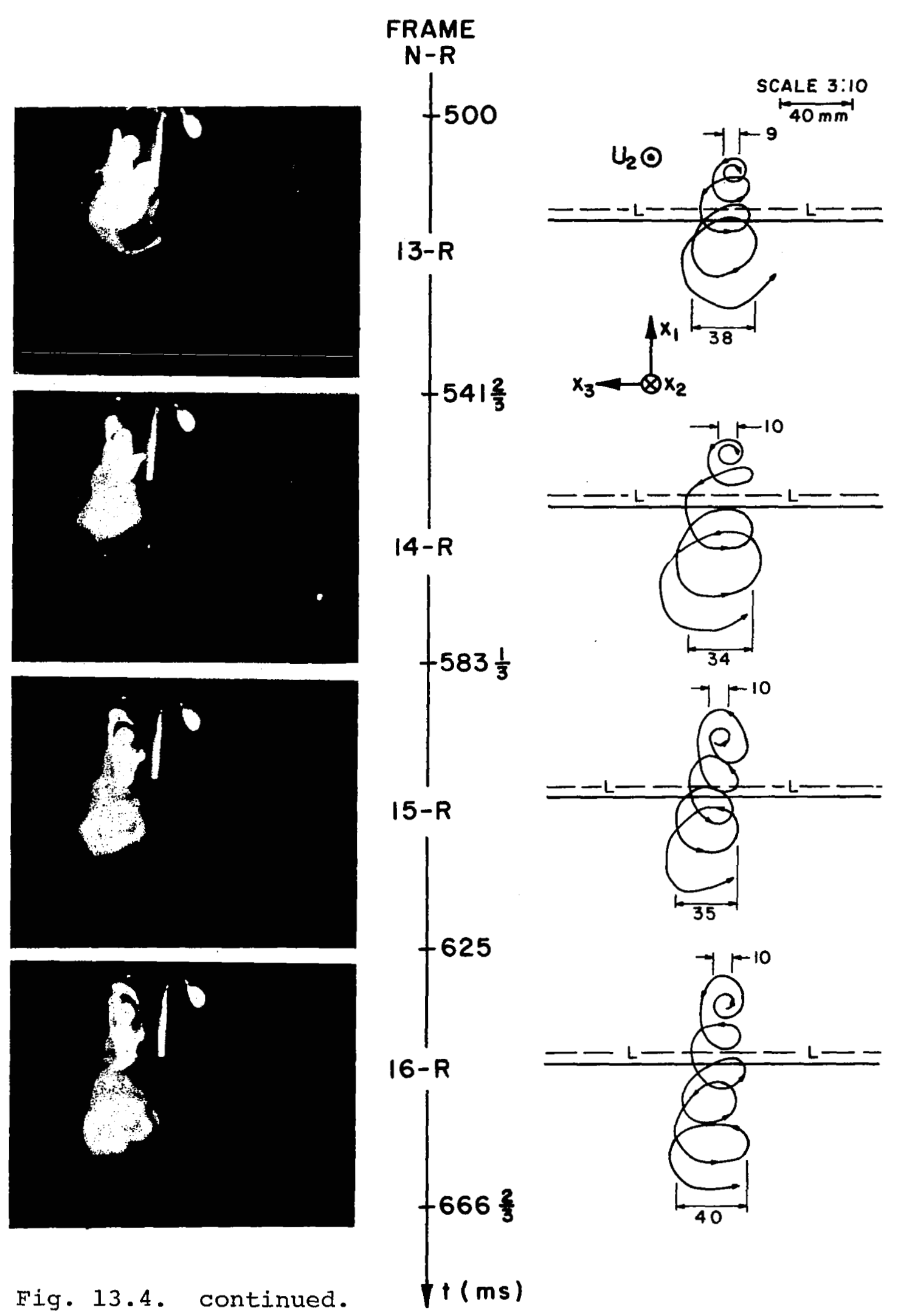




\begin{tabular}{|c|c|c|c|c|}
\hline $\begin{array}{l}\text { 1. Report No. } \\
\text { NASA CR-3019 }\end{array}$ & \multicolumn{2}{|c|}{ 2. Government Accession No. } & \multicolumn{2}{|c|}{ 3. Recipient's Catalog No. } \\
\hline \multirow{2}{*}{\multicolumn{3}{|c|}{$\begin{array}{l}\text { 4. Title and Subtizle } \\
\text { A VISUAL INVESTIGATION OF TURBULENCE IN } \\
\text { STAGNATION FLOW ABOUT A CIRCULAR CYLINDER }\end{array}$}} & \multicolumn{2}{|c|}{$\begin{array}{l}\text { 5. Report Date } \\
\text { October } 1978\end{array}$} \\
\hline & & & \multicolumn{2}{|c|}{ 6. Performing Organization Code } \\
\hline \multicolumn{3}{|c|}{$\begin{array}{l}\text { 7. Author(s) } \\
\text { Willy Z. Sadeh and Herbert J. Brauer }\end{array}$} & \multicolumn{2}{|c|}{$\begin{array}{l}\text { 8. Performing Organization Report No } \\
\text { CSU-FMWEP-NASLRC-1 } \\
\text { (CER 77-78WZS-HJ1322) }\end{array}$} \\
\hline \multirow{3}{*}{\multicolumn{3}{|c|}{$\begin{array}{l}\text { 9. Performing Organization Name and Address } \\
\text { Fluid Mechanics and Wind Engineering Program } \\
\text { Department of Civil Enginēering } \\
\text { Colorado State University } \\
\text { Fort Collins, Colorado } 80523\end{array}$}} & \multicolumn{2}{|l|}{ 10. Work Unit No. } \\
\hline & & & \multicolumn{2}{|c|}{$\begin{array}{l}\text { 11. Contract or Grant No. } \\
\text { NSG-3127 }\end{array}$} \\
\hline & & & \multirow{2}{*}{\multicolumn{2}{|c|}{$\begin{array}{l}\text { 13. Type of Report and Period Covered } \\
\text { Contractor Report }\end{array}$}} \\
\hline \multirow{2}{*}{\multicolumn{3}{|c|}{$\begin{array}{l}\text { 12. Sponsoring Agency Name and Address } \\
\text { National Aeronautics and Space Administration } \\
\text { Washington, D.C. } 20546\end{array}$}} & & \\
\hline & & & \multicolumn{2}{|c|}{ 14. Sponsoring Agency Code } \\
\hline \multicolumn{5}{|c|}{$\begin{array}{l}\text { 15. Supplementary Notes } \\
\text { Final report. Project Manager, Peter M. Sockol, Fluid Systems Component Division, NASA } \\
\text { Lewis Research Center, Cleveland, Ohio } 44135 \text {. Film supplement C-288 available on request. }\end{array}$} \\
\hline \multicolumn{5}{|c|}{ 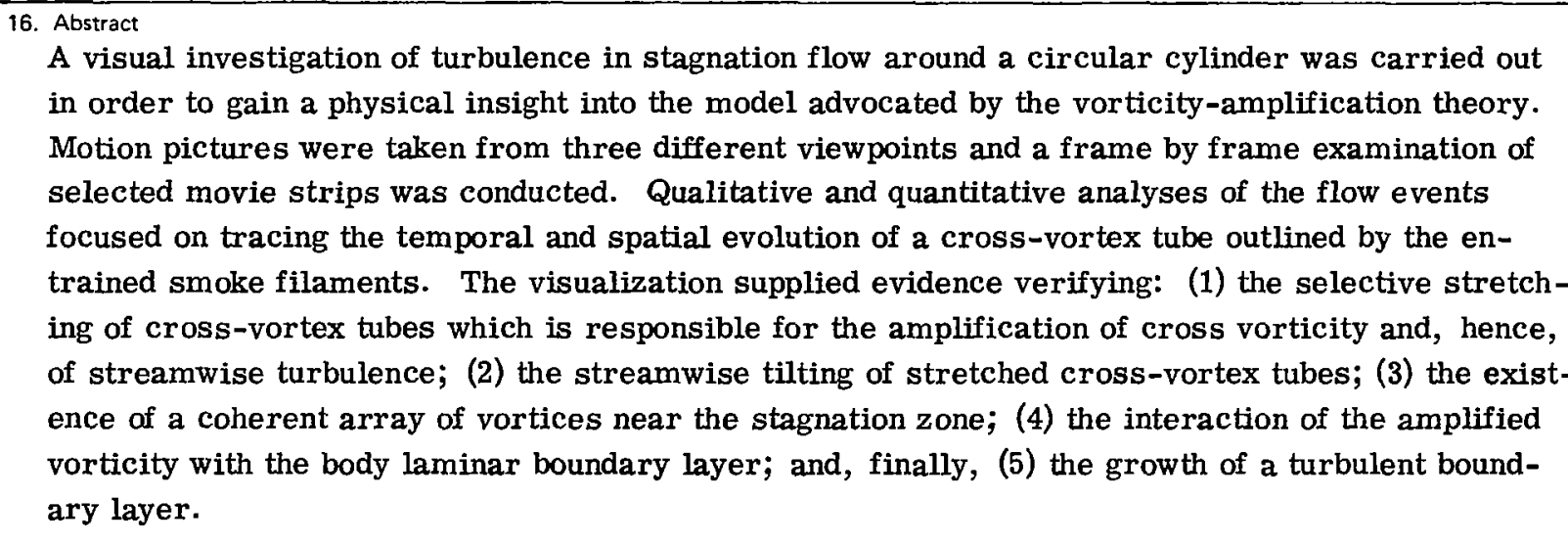 } \\
\hline \multicolumn{2}{|c|}{$\begin{array}{l}\text { 17. Key Words (Suggested by Author(s)) } \\
\text { Stagnation flow; Turbulent flow about a bluff } \\
\text { body; Vorticity amplification; Turbulence } \\
\text { amplification; Turbulent boundary layer; } \\
\text { Laminar boundary layer; Flow visualization }\end{array}$} & \multicolumn{3}{|c|}{$\begin{array}{l}\text { 18. Distribution Statement } \\
\text { Unclassified - unlimited } \\
\text { STAR Category } 34\end{array}$} \\
\hline 19. Security Classif. (of this report) & \multirow{2}{*}{\multicolumn{2}{|c|}{$\begin{array}{r}\text { 20. Security Classif. (of this page) } \\
\text { Unclassified }\end{array}$}} & 21. No. of Pages & 22. Price" \\
\hline Unclassified & & & 60 & $\mathrm{~A} 04$ \\
\hline
\end{tabular}

${ }^{*}$ For sale by the National Technical Information Service, Springfield, Virginia 22161 